Manche bevorzugen di e galvanokaustische Schlinge (\$243)

Glühschlinge vor der kalten, wie es scheint aus Furcht vor der Blutung. Vermuthlich ist diesen die vorzüglich arbeitende kalte s c h neidende Schlinge nicht bekannt, und sie benutzen eine nicht in die Führungsröhre einziehbare, lediglich quetschende Schlinge. Mit einer solchen kann man freilich keine etwas derbere Geschwulst glatt abtragen, man muß reißen und rupfen und kann danach unberechenbare Verletzungen und stärkereBlutungen hervorrufen. - Erwägen wir, daß diegalvanokaustische Schlinge trotz ihrer weit complicirteren Herrichtung und Anwendung, und trotz der sehr erschwerten Reinigung und Sterilisirung doch keine s i c h e r e Garantie gegen Blutungen gewährt, so werden wir ihr die kalte schneidende Schlinge für die Operation der entzündlichen Fibrome, wie überhaupt für die Mehrzahl der intranasalen Schlingenoperationen den Vorzug geben müssen.

Die galvanokaustische Schlinge wird im übrigen ebenso angelegt, wie die kalte. Beim Zusammenschnüren aber wird der Contact geschlossen, wodurch der bis dahin kalte Draht ins Glühen geräth. Durch häufiges Unterbrechen des Contactes kann man erreichen, daß möglichst zahlreiche durchschnittene Gefäßlumina mit dem rothglühenden Draht in Berührung kommen und dadurch verschlossen werden.

\title{
42. Kapitel \\ Die mit Atrophie einhergehenden Entzündungen der Nasenschleimhaut
}

\section{Ozäna ${ }^{1139-41}$}

Indem wir uns zur Besprechung der Ozäna wenden, betreten wir ein vielumstrittenes und äußerst unsicheres Gebiet, und zahlreich und bedeutend sind die Scbwierigkeiten, die sich uns entgegenstellen.

Die Ursache dafür liegt besonders in dem Umstande, daß das Krankheitsbild der Ozäna zu keiner Zeit festgestanden hat und auch heute noch nicht feststeht. Ja es hat den Anschein, als ob neue Forschungen eher dazu beitragen sollen, es zu verwirren als zu klären. Wollen wir deshalb die Autoren um ihre Meinung fragen, so müssen wir uns immer erst in das Krankheitsbild hineinleben, das sie sich von der Ozäna gemacht haben, und müssen versuchen, hieraus das für die Beantwortung unsrer Fragen Geeignete herauszusuchen oder zu erschließen. Daß dabei schiefe Urtheile und Irrthümer vorkommen können, ist leicht erklärlich, aber auch durch die Umstände einigermaßen entschuldbar.

Definition. Als Ozäna ${ }^{1142}$ bezeichnen wir eine Definitiou eigenthümliche diffuse Erkrankung der Nasenschleimhaut, diedurch Production ejnesdicken, z ur Krusten- und Borkenbildung tendirenden, spezifiseh übelriechenden Secrets und dureh

1139. KRIEG: Rhinitis atrophicans foetida (Ozaena) und non foetida. Heymanns Handb. 3. 1900. 1140. Grosskopff: Die Ozäna. Haugs Samml. V, 5. 1902. 1141. SсhönemanN, A.: Die Ozäna. Sammelref. Cbl. f. O. 1, S. 283.1903.

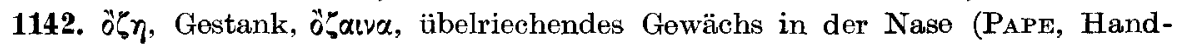
wörterb. d. griech. Sprache). 
Atrophie der Schleimhaut und des darunter liegenden $\mathrm{Nasenger}$ ästes charakterisirt ist.

Durch diese Definition sind vom Begriffe der Ozäna alle Gestank erzeugenden ci r c u s c r i t e $\mathbf{n}$ Erkrankungen des Naseninnern ausgeschlossen, also alle geschwürigen Processe und Knochennekrosen, wodurch sie auch erzeugt sein mögen, ferner die Nebenhöhlenempyeme und Rachenmandelaffectionen.

Symptome. Das hervorragendste und in unbehandelten Fällen 390 stets vorhandene Symptom der Ozäna ist ein $\ddot{\mathbf{u}}$ b l e r G e r u c h d e r symptome Exsti r pat i o $\mathrm{ns} l$ u $\mathrm{ft}$, der in leichten Fällen nur in unmittelbarer Nähe des Patienten bemerkbar zu sein braucht, sich aber bei vollentwickelter Krankheit zu einem entsetzlichen, die Umgebung des Kranken in weitem Umkreise verpestenden Gestanke steigern kann. Es ist schwer, die Abscheulichkeit dieses Gestankes mit Worten zu Fotor schildern. „, Sie riecht aus dem Halse, als ob sie ihre Füße verschluckt hätte“", läßt ZoLA (L'assommoir) ein Fabrikmädchen von einer andern sagen, neben der sie nicht sitzen will. Selbst dem, der durch bäufigen Verkehr mit Ozänakranken einigermaßen abgestumpft ist, kommt oft ein Brechreiz an, wenn er beim Ausräumen der Borken den Fötor aus nächsteı Nähe einathmen muß.

Über den $\mathrm{Charakter} d$ es Fötors sind die Meinungen getheilt. Für mich hat er, wie für viele andre ${ }^{1143}$ etwas durchaus Specifisches an sich, das ich sonst so gut wie nirgends gerochen habe. (Nur nach Zerstörung des Naseninnern durch tertiäre Syphilis findet man manchmal einen ähnlichen Fötor. Aber hierbei sind auch die anatomischen Veränderungen denen bei der Ozaena vera sehr ähnlich.) Mich erinnert er nicht an den Geruch zerquetschter Wanzen (nach einigen soll das französische Wort für die Stinknase, punaisie, von punaise abgeleitet sein), sondern viel eher an Schweißfüße (ZoLA) oder an fötide Cholesteatome. - Andre 791. 806. 1139/51 wiederum vermögen am Ozänagestank keine eigenthümliche Färbung wahrzunehmen. - Zur Erklärung solcher Differenzen muß man auf Anomalieen der Geruchsperception bei der einen von beiden Parteien verweisen oder daran denken, daß die Einen die Grenzen des Ozänabildes anders ziehen als die Andern.

Frese 1150 fand bei der chemischen Untersuchung des Ozänasecrets stark alkalische Reaction, von übelriechenden Bestandtheilen Indol und Skatol, Phenol,

1143. z. B. Löwenkerg ${ }^{1144}$, Walb ${ }^{1145}$, Jurasz ${ }^{794}$, SChech ${ }^{805}$, Flatad ${ }^{780}$, GOTTSTEIN 1146, RÉTHI 1147, CHOLEWA 1148, Gerber 1149. 1144. Löwenkerg: Die Natur und die Behandlung der Ozäna. D. m. W. 1885, 1/2. 1145. WaLB: Erfahrungen a. d. Gebiete d. Nasen- u. Rachenkrankh. 1888.1146. Gotsstein, J.: Zur Pathologie u. Therapie d. Ozäna. Bresl. ärztl. Zeitschr. 1879, 17/18. 1147. REthI: Z. Wesen u. z. Heilbarkeit d. Ozäna. A. f. L. 2. 1895. 1148. Cholewa \& Cordes: Zur Ozänafrage. ibd. 8. 1898. 1149. Graber: Chamäprosopie und hereditäro Lues in ihrem Verh. zur Platyrrhinie u. Ozäna. ibd. 10. 1900. 1150. Frese, O. (Halle a. S.): Unters. üb. Entstehung und Wesen d. Fötors bei Ozäna. D. A. f. kl. Med. 86. 1905. 1151. Demme: U̇ber Ozäna. 
Schwefelwasserstoff, besonders reichlich aber flüchtige Fettsäuren, diese, wie er annimmt ( $\$ 405$ ) nicht durch Eiweißfäulniß, sondern durch directe Umwandlung neutralenFettes (aus fettig degenerirten Infiltrations- und Drüsenzellen) entstanden.

Die Stärke des Gestankes kann schwanken, bei Frauen manchmal mit den Menstruationsvorgängen ${ }^{\mathbf{7 9 4}}$.

Bei der äußeren Betrachtung fällt zuweilen eine b e s o n d e r e Gestalt der $\mathrm{Nas}$ e auf ${ }^{1139^{/ 51}-53}$. Sie ist abgeplattet, breit und im Verhältniß zum übrigen Gesicht im Ganzen klein, die Nasenlöchrer sehen etwas mehr nach vorn als gewöbnlich, d. h. wir haben die Form der Sattelnase vor uns, ähnlich wie sie bei hereditärer Lues gefunden wird ${ }^{1149}$.

Die rhinoskopische Besichtigung zeigt die Nasenwände bedeckt mit klebrig-schmierigen, stellenweise festeren, grüngel ben oder schmutziggrauen oder schwarzgescheckten Borken, Schalen,

secret Krusten oder Klumpen. Die Massen sitzen an einzelnen Stellen fester auf ihrer Unterlage, im übrigen findet man zwischen ihnen und der Schleimhautoberfläche eine dünne glasige oder gelbgrüne Eiterschicht, sodaß sie hier mühelos abgehoben werden können. Die Krusten lassen entweder noch ein geringes Lumen für die Luftpassage frei oder sie verstopfen die Nase vollends. Aus ihrer Masse darf kein Rückschluß auf die Abundanz der Secretion gemacht werden. Die täglich abgesonderte Secretmenge ist nicht sehr bedeutend und nur dem Umstande, daß das Secret tagelang in der Nasenhöhle liegen bleibt, verdanken die Borkenmassen ihre Mächtigkeit ${ }^{800.1139 / 48}$.

Ähnlich wie die Nasenhöhle ist auch der Nasenrachen mit Borken und Secretklumpen ausgeklebt. Sie ziehen sich manchmal unter dem Gaumensegel hervor noch eine Strecke weit in den Mundrachen hinab.

An den Borken haftet der vorhin geschilderte mephitische Gestank. Nach ihrer Entfernung ist die Nase ohne üblen Geruch. Auch das frisch abgesonderte Secret ist völlig geruchlos ${ }^{1146 / 55 / 56}$ - nur JURASz ${ }^{794}$ behauptet das Gegentheil --, der Gestank stellt sich darin also erst später ein, während das Secret stagnirt und sich eindickt.

Bei der mikroskopischen Untersuchung des Secrets findet man von morphologischen Bestandtheilen vorzüglich Eiterzellen und äußerst zahlreiche Mikroorganismen der verschiedensten Arten vor (Figg. 42. 43. $\S 401$ ). SCHUCHARDT ${ }^{115} 7$ gibt an, daß daneben zahlreiche z. Th. kernlose Plattenepithelien vorhanden seien.

Die $F$ a r bedes $S$ ecrets und der Borken ist von zwei Ursachen abhängig. Erstens von fremden Beimengungen, die entweder mit der Inspirations-

Berl. laryngol. Ges. Verh. Bd. 2. 1892. 1152. ZaUFAL: Üb. d. Anomalieen in der Bildung der Nasenmuscheln. Ärztl. Corresp. -Bl. f. Böhmen. 3. 23.1875. 1153. HabermanN: Zur patholog. Anat. d. Ozaena simplex s. vera. Zeitsehr. f. Heilk. 7. 1886. 1154. Steiner, MaximiL.: Z. Weiterentwickelung d. Lehre v. d. Ozäna. A. f. L. 21, S. 282 . 1908. 1155. FräNKEL, E.: Weitere Unters. üb. d. „Rhinit. chronica atroph. foetida“. Virch.s Arch. 90. 1882. 1156. KUTTNER: DieTherapie der Ozäna. Therapeut. Monatsh. 1893. März. 1157. ScHdChardT: Üb. d. Wesen d. Ozäna. Volkmanns Samml. 340. 1889. 
luft hineingelangen (Staub, Ruß u. dgl.) oder von der Schleimhaut geliefert werden (Blutfarbstoff und dessen Umwandlungsproducte). Zwoitens von bakteriellen Zersetzungen innerhalb der Secretschicht. Daher stammt die Gelb- und Grünfärbung der Secrete.

Muck 1158 findet, daß Rhodan, ein constanter, aus den serösen Drüsen stammender Bestandtheil des normalen, des katarrhalischen und des eitrigserösen Nasensecrets, im Ozänasecret nicht vorhanden ist ( $\$ 61)$.

Erst nach der Entfernung der Borken können wir uns von der 392 Gestalt der $\mathrm{Nasenh}$ öhle ein rechtes Bild machen (vergl. Atrophie Fig. 121). Wir finden sie stets abnorm groß und zwar vorzugsweise

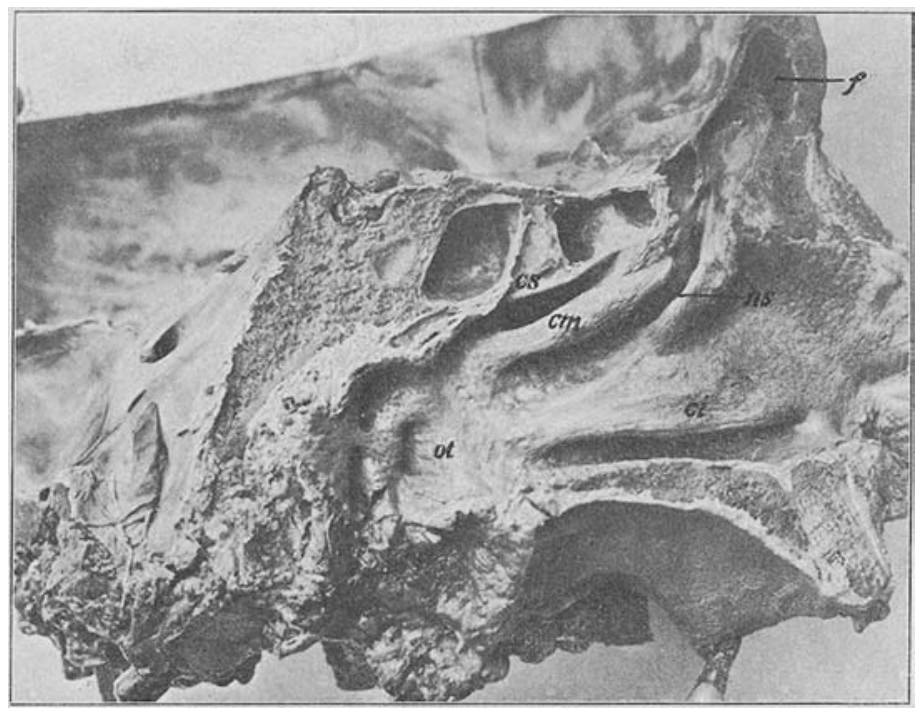

Fig. 121. Linke Nasenhälfte. Atrophie der Nasenschleimhaut und der Nasenknochen (A. Hartmanns Sammlung).

ci, cm, es untere, mittlere, obere Muschel. f Sinus frontalis. hs Hiatus semilunaris. ot Tubenmündung.

durch Schwund der Nasenmuscheln, besonders der unteren. An Stelle dieser finden wir oft nichts anderes, als eine Schleimhautfalte, die wie ein Zeltdach den unteren Nasengang überdacht. Ist dabei die mittlere Muschel intact, so erscheint sie besonders groß. Manchmal aber ist auch sie fast völlig eingegangen, sodaß die Gegend des Hiatus semilunaris freiliegt. Nicht selten kann man durch die erweiterte Riechspalte die vordere Keilbeinfläche und die Keilbeinhöhlenmündungen übersehen und unter der Leitung des Auges sondiren. Ausnahmsweise ist auch die dritte Muschel sichtbar ${ }^{799}$. Besonders deutlich aber präsentirt sich die Hinterwand des Nasenrachenraumes, die Tubenmündung mit ihrer Umgebung; und das Spiel der Tubenmuskulatur beim Sprechen,

1158. Muck: Weiteres über das Vorkommen von Rhodan im Nasensecret und über das Fehlen von Rhodan bei der Ozäna. Z. f. O. 38, S. 321.1901. 
Schlingen und Würgen läßt sich bei solchen Fällen mit vortrefflicher Klarheit studiren ( $\$ 85$ ).

Zuweilen ist bei Atrophie der untelen Nasenmuschel die Schleimhaut der oberen Nasengegend nicht nur nicht verdünnt, sondern eher aufgelockert und verdickt trotz Atrophie des darunterliegenden Knochens und so kann es vorkommen, ,da an einem geringen Muschelknochenrest dicke, in Runzeln gelegte Schleimhautlappen baumeln, etwa so wie bei Pädatrophie die zu weit gewordene Haut den Knochen des fettund muskelarmen Schenkels umschlottert" (KRIEs 1139, s. 417).

Im Übrigen erscheint die Schleimhaut im Ganzen blaß, sehnenähnlich. Mit der Sonde vermag man sie nicht oder nur sehr wenig einzudrücken. Dagegen fïndet man die knöcherne Unterlage abnorm weich ${ }^{1148}$ und noch mehr reducirt als den Schleimhautüberzug.

Gewöhnlich sind beide Nasenhälften gleichzeitjg ergriffen. Es soll aber auch, obwol sehr selten, eine Seite allein erkranken können (Oza ena unilateralis).

Bei der Betrachtung mit dem Rachenspiegel Nasen- tindet man den $\mathrm{N}$ a senrachen übermäßig weit ${ }^{1159}$. Die Errachen weiterung beruht $z$. Th. auf abnormer Dünne der Schleimhaut und auf Schwund des lyniphadenoiden Polsters, z. Th. aber, wie Hopmans 1160/62 durch Messungen festgestellt hat, auf einer in der Configuration des Skeletts begründeten Vergrößerung des sagittalen Durchmessers und entsprechender Terkürzung d es Septu ms.

Hopmann maß zuerst die Entfernung der Nasenspitze von der Rückwand Septum des Nasenrachens (a), sodann die Entfernung der Nasenspitze vom hinteren Rande des Septums (b). Daraus erhielt er die Tiefe des Nasenrachens gleich $a-b$. Um die Werthe für Vergleichungen geeignet zu machen, wurden sie auf die Zahl 100 für a umgerechnet. Auf diese Weise fand H. bei 40 Ozänakranken den Durchschnittswerth für das Septum kleiner als 71, die Nasenrachentiefe dementsprechend größer als 29; bei 61 Kranken mit andern Nasenaffectionen oder Gesunden das Septum größer als 77 und die Nasenrachentiefe dementsprechend kleiner als 23. - Die Messungen Hopmanns sind von Gerber ${ }^{1149}$ bestätigt, von GR ÜNWALD ${ }^{1161 / 63}$ dagegen, wie mir scheint ohne hinreichende Begründung, angefochten worden.

Die in den Messungen nachgewiesene abnorme Kürze des Septums war in den Fällen Hopmanns nicht durch Randnekrose des Vomers verursacht, sie beruhte vielmehr darauf, daß die ganze Choanenebene nach vorn verschoben war. Daraus schließt H., daß es sich um einen hereditären, in der Anlage bereits

1159. Die Weite des Nasenrachens und die Gestalt seiner Wände, besonders auch der Choanen und des hinteren Vomerrandes, lassen sich vortrefflich an Abdrücken studiren, die nach dem Verfahren Hopmanns (Z. f. L. 1, S. 305. 1908) mit Stents Maße hergestellt werden. 1160. Hopmass: Über Messungen des Tiefendurchmessers der Nasenscheidewand bezw. des Nasenrachenraumes; ein Beitrag zur ätiologischen Beurtheilung der Ozäna. A. f. L. 1. 1894. 1161. GRünwald: Weitere Beiträge zur „Ozäna"-Frage. M. m. W. 1893, 43/44. 1162. HopmanN: Ozaena genuina. M. m. W. 1894, 3. 1163. Grünwald : Altes und Neues über die Stinknase. M. m. W. 1894, 15. 
bei der Geburt vorhandenen Hemmungsproceß, um eine Entwickelungsstörung handele, die analog andern ähnlichen Vorgängen häufig erst während der Pubertätsperiode manifest werde.

SiebenmanN ${ }^{1164}$ hält die Kürze des Septums für eine Theilerscheinung der Chamäprosopie ( $\$ 109$ ), der er, wie wir hören werden, eine bedeutende Rolle für die Entstehung der Ozäna zuerkennt.

Das Ger u chs verm ö gen ist gewöhnlich herabgesetzt oder 394 vernichtet 1165-67. Die Anosmie soll zuweilen eine respiratorische, Anosmie durch Verklebung der Riechspalte oder durch Ablenkung des Luftstromes erzeugte sein, in der Regel aber liegen ihr degenerative Veränderungen der Riechschleimhaut, insbesondere des Riechepithels zu Grunde ( $\$ 400)$, sie ist also eine essentielle Anosmie.

Sie macht es erklärlich, daß Ozänakranke im Gegensatze zu dén mit fötiden Nebenhöhlenaffectionen Behafteten von ihren Exhalationen selten belästigt werden. Dagegen macht der Gestank sich der Umgebung höchst unangenehm bemerklich (§ 390) und veranlaßt Angehörige oder Bekannte, den Kranken in zarter oder auch unzarter Weise davon Mittheilung zu machen. Die Quelle des Gestanks wird dabei gewöhnlich in den Hals oder in den Magen verlegt. Welchen Eindruck eine derartige Mittheilung auf ein empfindliches Gemüth machen muß, ist leicht zu ermessen. Die Kranken sehen sich durch ihre Exhalationen geächtet, um so mehr als ihnen die Controlle durch den eigenen Geruch fehlt, sie ziehen sich vom Verkehr zurück, werden menschenscheu, oft schwermüthig und lebensüberdrüssig.

Viele klagen über das $G$ ef ühl von Trockenheit in der Nase und im Halse.

Selten fehlen $K$ opf $\mathrm{druck}$ und $\mathrm{K}$ opf schmerzen. Sie können von der Resorption der verdorbenen Inspirationsluft, von der zeitweiligen Nasenverstopfung, von der mit heftigem Schnäuzen verbundenen Blutstauung, endlich von einer complicirenden Nebenhöhlenerkrankung abhängen.

Complicationen. Zuweilen werden spontane $\mathrm{Nasen}$ b I u t u n g e $\mathrm{n}$ beobachtet. Vermuthlich handelt es sich um Personen, die unabhängig von ihrer Ozäna an Nasenblutungen leiden. Aber es läßt sich auch verstehen, daß die z ur Elimination der Secrete unternommenen Anstrengungen das Nasenbluten befördern.

Um die Borken loszuwerden, müssen die Patienten nämlich lange und heftig schnauben oder die Luft heftig nach hinten durchsaugen.

1164. Simbenmanv: $\dot{\mathrm{U}} b$. adenoiden Habitus and Leptoprosopie, sowie üb. das kurze Septum der Chamaeprosopen. M. m. W. 1897, 36. 1165. Morf: Ein Beitrag z. Symptomatologie d. Rhinit. chron. atrophica mit bes. Beruicksichtigung der Affectionen des Gehörgangs. Z.f. O. 25. 1894. 1166. ZwaArdemaker: Physiologie und Pathologie des Geruchs. 68. Vers. Deutseher Naturf. a. Ärzte. Frankf. 1896. ref. Semons Cbl. 13, S. 120. 1167. Reuter: Essentielle Anosmie. A. f. L. 9.1899. 
Bei Lockerung des Nasenrachensecrets kann es dann leicht z u W ü r g e n o d e r E r b rech e n kommen. Zuweilen werden Secretklumpen verschluckt.

In manchen Fällen sind die $t$ i ef e r e $n ~ L u f t w$ e ge afficirt, entweder in Form des einfachen oder des sogenannten trockenen chronischen Katarrhs ( $P$ haryngit is, La ry $\mathrm{n}$ it is, Tracheit is chronica sicca, Ozaena laryngis et trachae). Im Mundrachen kommt es dabei nicht zur Ansammlung von trockenen Borken, denn beim Schlucken wird das Secret jedesmal gelockert und entfernt. Dagegen kann man im Kehlkopf und Luftröhre beträchtliche, Athemmangel verursachende Klumpen fötiden Secrets wahrnehmen.

AleXANDer ${ }^{1168}$ hat unter 50 Ozänafällen 22 unzweifelhafte und 7 suspecte Fälle von $\mathrm{L} u \mathrm{n}$ g e $\mathrm{n} \mathbf{t} \mathrm{u}$ b e $\mathrm{rk}$ u l o s e gefunden, was dafür spricht, daß die Ozäna eine Disposition für diese Erkrankung abgibt.

Die Betheiligung der Nasennebenhöhlen bei der Ozäna werden wir später noch zu besprechen haben.

Über die Betheiligung des Mittelohrs gehen die Angaben auseinander. MTCHEL ${ }^{799}$ und LöWENBERG ${ }^{1144}$ finden sie sehr selten, JURAsz ${ }^{794}$ beobachtete sie bei 170 Fällen 12 mal, ZaUFAL ${ }^{1169}$ in 80 pct. seiner Fälle. MoRF 1165 constatirte an einem Material von 80 Fällen 47,5 pct. Ohraffectionen überhaupt, wobei das Mittelohr 4 mal so häufig erkrankt war wie das innere Ohr.

Nach HeYmans 1170 und KaYsER ${ }^{1171}$ schließen Ozäna und adenoide Vegetationen einander aus.

Ozaena

öfters noch eine zweite Form der Ozäna angenommen, die o h n e Atrophi $\theta$ einhergeht. Bei ihr bietet die Schleimhaut die Veränderungen des chronischen Katarrhs, während das Secret dem bei der Ozäna ähnlich sein soll. Bei der Betrachtung solcher Fälle kann man sich des Verdachts nicht erwehren, es möchten Verwechselungen mit den foetiden Nebenhöhlenempyemen vorliegen.

Vorkommen und Verlauf. Seltener erkranken wolgenährte und. blühende Personen an Ozäna. Gewöhnlich findet man diese bei schlechtgenährten blutarmen Indïviduen mit blasser, fahler Hautfarbe, schlaffer Muskulatur, geringer Kraft und Energie. Viele fassen diese Constitution als das Primäre auf und meinen, daß sie zur Ozäna disponire. Andere leiten die schlechte Ernährung als secundäre Erscheinung von den Schädigungen der Nasenerkrankung, insbesondere der unausgesetzten Einathmung des eigenen Gestankes,

1168. Alexander: Die Beziehungen der Ozäna zur Lungentuberkulose. A. f. L. 14. 1903. 1169. Z Z AUFAL: Ửb. d. allgem. Verwendbark. d. kalten Drahtschlinge etc. Prager medic. Wschr. 1877. Sep.-Abdr. 1170. HeyManN: B. lar. Ges. 10. 6. 04. Verh. Bd. 15, S. 36. 1171. Kayser (Breslau): İb. d. Verhältniß d. Ozäna zu d. adenoiden Vegetationen. 68. Vers. D. Naturf. u. Ärzte. Frankfurt 1896. ref. Semons Cbl. 13, S. 115 (ausf. publicirt. in d. Wiener klin. Rundschau. 1897, 9). 
von dem zeitweiligen Verschlucken der Secrete und den dadurch erzeugten Verdauungsstörungen ${ }^{799}$, endlich von der psychischen Depression solcher Patienten ab. Vermuthlich haben beide Anschauungen ihre Berechtigung.

Die Erkrankung wird, wie nach dem Gesagten plausibel erscheinen muß, öfter bei den ärmeren als bei den wohlhabenden Bevölkerungsschichten angetroffen.

Sie befällt etwa doppelt ${ }^{794.1139}$, nach GERBER ${ }^{1149}$ und STEINER ${ }^{1154}$ sogar fast dreimal so häufig das weibliche wie das männliche Geschlecht.

Mehrmals hat man Häufung bei nahen Verwandten beobachtet $1144 / 72-75$. Es kann sich dabei um eine Vererbung der Disposition handeln oder, wie die Anhänger einer bacillären Entstehung wollen, um directe U̇bertragung.

Die meisten Fälle kommen erst nach Beginn der Pubertät zur Be- 397 obachtung ${ }^{1139}$, doch beginnt die Krankheit gewöhnlich schon früher ${ }^{1176}$. Verlauf Sie entwickelt sich unmerklich, schleichend. Sie wird gewöhnlich anfangs für einen hartnäckigen Schnupfen angesehen und die Kranken suchen den Arzt erst auf, wenn sie voll ausgebildet ist. So kommt es, daß nur ganz vereinzelte Beobachtungen über das Aussehen des Naseninnern vor Eintritt der Erkrankung existiren.

Wenn wir Ozänakranke nach der Entstehung ihres Leidens fragen, so antworten sie ungefähr Folgendes: Sie litten schon lange Zeit an Schnupfen, d. h. an vermehrter Absonderung aus der Nase. Das Abgesonderte wäre allmählich dicker geworden, hätte Mühe beim Ausschnauben gemacht und hätte einen üblen Geruch angenommen, der immer stärker geworden sei. Von anfänglicher Nasenverstopfung wissen viele nichts. Manche geben an, daß sie zeitweilig eine solche bemerkt hätten.

Aus solchen Angaben läßt sich nichts weiter schließen, als daß die Ozäna sich schleichend entwickelt und daß sie ein geruchfreies Vorstadium hat. Welcher Art aber dieses Vorstadium ist, welche Veränderungen an der Schleimhaut und dem Secret dabei im Spiele sind, entzieht sich völlig unserm Urtheil.

Demgegenïber steht eine andre weitverbreitete Ansicht, wonach $\mathrm{der}$ Ozäna regelmäßig ein hypertrophischor Katarrh vora u s g e e n s olle. Beide Zustände seien nichts weiter als verschiedene Stadien desselben Processes, des chronischen Katarrhs. Deshalb hat Gortscers 1177 die Krankheit als Rhinitis chronica atrophicans foetida bezeichnet und diese Bezeichnung ist fast allgemein acceptirt worden.

1172. Rosenfeld: Ätiologie d. Ozäna. 10. intern. med. Congr. Berlin 1890. Verh. Bd. IV, 12. 1173. StrüBING: Über Ozäna. M. m. W. 1895, 39/40. 1174. Cholewa bei Cholewa \& Cordes 1148. 1175. Perez, F.: État actuel de la question „Ozène“ etc. Ann. des mal. de l'oreille etc. 33, 6. 1907. 1176. Treitel: In welchem Alter zeigt sich zuerst die Ozäna ? A. f. L. 16, S. 336. 1904. 1177. Gottstein: B. kl. W. 1878, 37. cit. von demselben ${ }^{1146}$. 
Für die Entscheidung der vorliegenden Frage sind an tomische Untersuchungen leider völlig werthlos. Daß neben atrophischen Schleimhautbezirken in der Ozänanase auch einmal hypertrophische vorkommen, kann man klinisch ebenso gut wie anatomisch feststellen. Aber damit ist nichts gewonnen. Worauf es ankommt, das sind fortlaufende, genaue Beobachtungen, welche lehren, daß bei demselben Individuum die Verdickung der Nasenschleimhaut mit dem Auftreten der Ozäna in Verdünnung übergeht. Solche Beobachtungen existiren bisher nicht. Ein einzelner Fall von SchÄFFER ${ }^{1178}$ ist, wie bereits MoLdenHaUte ${ }^{800}$ hervorgehoben hat, deshalb nicht einwandfrei, weil es sich um ein hereditär syphilitisches Individuum gehandelt hat. Dagegen haben RÉтнг ${ }^{1147}$ und CHоLewa ${ }^{1148}$ die Entstehung von Ozäna ohne vorhergehende Nasenschleimhauthypertrophie beobachtet.

Die Ozäna zeigt in der Regel einen Wechsel in der Stärke der Symptome, Exacerbationen und Remissionen lösen einander ab. In einzelnen Fällen sind, besonders bei älteren Personen, sogar spontane andauernde Besserungen beobachtet worden, ja selbst Ausheilungen in dem Sinne, daß die Patienten ohne Behandlung des Leidens andauernd

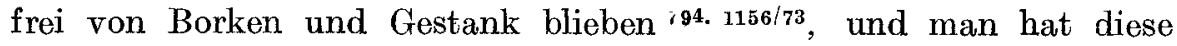
Veränderungen auf völlige Vernichtung der secernierenden Apparate, insbesondere der Drüsen zurückgeführt. In der Regel dürfte aber das Leiden dauernd bestehen bleiben, wenn ihm nicht eine zweckentsprechende Behandlung zu Theil wird.

Pathologische Anatomie. Bakteriologie. Zuokerkands' hat an Leichenpräparaten die näheren Vorgänge bei der Atrophie der unteren Muscheln verfolgt. Er berichtet darïber: „Die Muschel wird vorerst dünner, biegsamer und kleiner, u. z. sowol im Knochen, als auch in der Schleimhaut; später wird sie auch flacher, und so schreitet der Proceß langsam vorwärts, bis man schließlich im höchsten Grade der Atrophie an jener Stelle der äußeren Nasenwand, wo ehemals die untere Muschel abging, nur mehr eine Schleimhautleiste vorfindet, in der zuweilen noch als Rest der Muschel ein Knochenstäbchen eingelagert ist. Die Schleimhaut schrumpft, wird gefurcht; der Schwellkörper der Muschel ist dabei geschwunden, und die blasse, dünne, glänzende Mucosa gleicht schließlich mehr einer Serosa als einer Schleimhaut." Am macerirten Muschelbeine zeigt sich ,im schwächsten Grade der Atrophie die untere Muschel bloß verdünnt, brüchiger, stellenweise auch schon perforirt; im weiteren Verlaufe der Atrophie vermindert sich die Höhe des Muschelbeines, der freie Rand ist nicht mehr convex, sondern gradlinig oder gar concav. Durch Dehiscenzen löst sich die Randzone in Form eines schmalen Bandes größtentheils ab, später schwindet auch diese Zone, neue bilden sich, schwinden abermals, bis schließlich vom Muschelbeine nur mehr ein Leistchen zurückgeblieben ist."

In einer Anzahl von Fällen sind die $\mathrm{N}$ e b e $\mathrm{n} h$ ö h $\mathrm{l}$ e $\mathrm{n}$ einzeln oder combinirt erkrankt gefunden worden. In einigen andern aber ist durch die Section jede Nebenhöhlenaffection sicher ausgeschlossen worden $117^{9-85}$.

1178. SchäFfer: Ozäna. M. f. O. 1881, Sp. 57. 1179. FränKel, E.: Beitr. z. Rhinopathologie. Virchows Arch. 87. 1882. (1 Fall.) 1180. Ders.: Weitere Unters. üb. d. „Rhin. chron. atrophica foetida". Virchows Arch. 90. 1882. (1 Fall.) 1181. Wertheim: Beitr. z. Pathol. u. Klinik d. Erkrank. d. Nasennebenh. A. f. L. 11. 1901. (2 Fälle.) 1182. Minder: 50 Sectionshef. d. Nase und deren Nebenhöhlen unter Berücksichtigung der Gesichtsschädletmaße. ibd. 12. 1902. (2 Fälle) 1183. Schönemañ: Die Um- 
Histologiseh $\Theta$ Untersuehungen ${ }^{1186}$ haben gezeigt, daß im 400 Anfange des Processes eine starke Rundzelleninfiltration der Schleimhaut vorhanden ist, dazwischen ist mehrmals Einlagerung massenhaften gelbbraunen Histologie

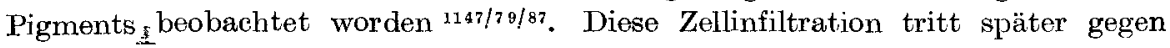

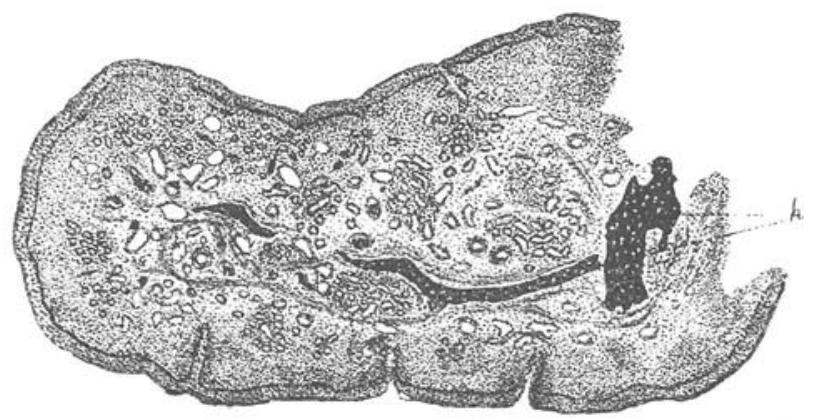

Fig. 122. Ozäna, mittlere Muschel. ${ }^{10} / 1$.

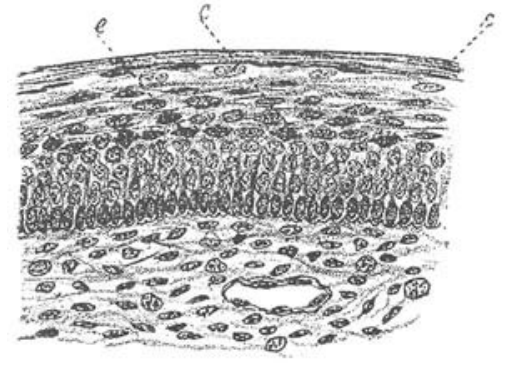

Fig. 123. Eine Stelle des Epithels von Fig. 122, $340 / 1$. c verhornte Zellen, e e Eleïdinzellen.

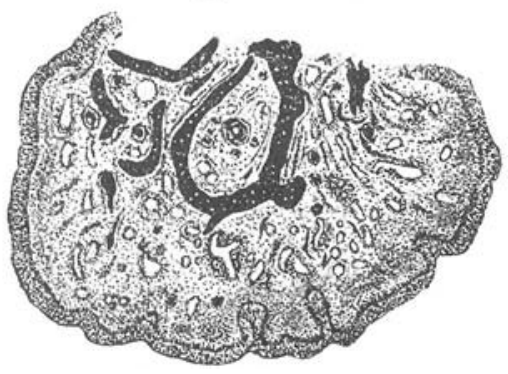

Fig. 124. Ozäna, mittlere Muschel. $10 \%$.

(Die Figg. 122-124 sind nach Präparaten gezeichnet, die ich in Fällen ausgesprochener Ozäna nach Abtragung von Stücken der mittleren Muschel gewonnen habe. Man erkennt die dicke subepitheliale Infiltrationszone. In Fig. 122 reichlich Drüsen, wenig Gefäße; das Cmgekehrte in Fig. 124. Regelrechtes Epithel zeigen nur wenige Bezirke, die meisten entweder Übergangsepithel oder verhornendes Pflasterepithel, das letztere in einem Falle mit deutlichem Stratum granulosum (Fig. 123), obwol die Präparation ( $\$ 192$ ) der Erhaltung des Eleidins nicht besonders günstig ist.)

wandlung (Metaplasie) des Cylinderepithels z. Plattenepithel i. d. Nasenh. d. Menschen und ihre Bedeutung f. d. Ätiol. d. Ozäna. Virchows Arch. 168. 1902. (1 Fall.) 1184. Dexte: Ansiedelung von Aspergillus fumigatus in beiden Nasenhöhlen bei Ozäna. Z. f. O. 46. 1904. (1 Fall.) 1185. OppIkofer: Beitr. z. normalen u. pathol. Anatomie d. Nase u. ihrer Nebenh. A. f. L. 19, S. 28. 1906. (2 Fälle.) 1186. GottsteiN 1146, KraUSe ${ }^{1187}$, E. FränKeL 1170/80, HaberMANN ${ }^{1188}$, SChUChardt ${ }^{157}$, SeIfert ${ }^{818.1189}$, ZarNiko ${ }^{813}$, Cholewa \& Cordes ${ }^{1148}$, Minder ${ }^{1182}$, Schönemann ${ }^{1183}$, OpPikofer ${ }^{1185}$, Streit ${ }^{1100}$. 1187. KraUSe, H.: Zwei Sectionsbefunde von reiner Ozäna. Virchows Arch. 85. 1881. 1188. HABERMaNN : Z. patholog. Anat. d. Ozaena simplex s. vera. Zeitschr. f. Heilk. Bd. 7. 1886. 1189. SeIFERT: Rhinit. atrophicans. 10. internat. medic. Congreß. Berlin 1890. Verh. Bd. IV, 12. 1190. Streit, H.: Histologisch-klinische Beitr. zum Sklerom. A. f. L. 16, S. 423. 1904. 1191. L. c. NoNo. 1180. 1187. 1157. 813. 1148. 1182-84. 1190. 1185 . 
die Neubildung faserigen Bindegewebes zurück, womit ein bedeutender Schwund von Drüsen und Gefäßen einhergeht. Das Epithel ist selten normal 1180, gewöhnlich ist es in ein kubisches oder mehrschichtiges Pflasterepithel umgewandelt, dessen oberflächlichste Schichten verhornen können 1191. Dabei kann as durch Vordringen des Stratum epitheliale nach der Schleimhaut zur Papillenbildung kommen 818, Tab.6. An den Knochenrändern sieht man zahlreiche HowsHIPsche Lacunen mit Osteoklasten darin, Osteoblasten fehlen oder sie sind sehr spärlich, was auf ein besonderes Überwiegen der Resorption ïber die Apposition der Knochensubstanz schließen läßt ${ }^{1148}$. In den Drüsenzellen ist fettige Degeneration beobachtet 1147/48/87/88, ebenso von Einigen ${ }^{1147 / 87}$ bei den infiltrirenden Leukocyten. Eine eigenthümliche schleimige Degeneration der Drüsenzellen beschreibt SohönEmaNn 1183. Gefäße und Nerven sind in der Regel unverändert.

Wir haben schon gesehen, daß das Ozänasecret zahlreiche M i k r o o r g a$\mathrm{n}$ i s $\mathrm{m}$ e $\mathrm{n}$ von den verschiedensten Arten beherbergt (Figg. 42. 43, S. 63). Es liegt die Frage nahe, ob sie eine wesentliche Rolle in der Krankheit spielen und welches diese Rolle ist.

Bakteriologische Untersuchungen 1192-96 haben gelehrt, daß eine ganze Anzahl derselben zufällig in die Secrete aspirirte, rein saprophytische Luftkoime ohne erwähnenswerthe Bedeutung sind, die deshalb von vornherein außer Betracht kommen. Andere Arten sind vereinzelt für das Zustandekommen des Ozänaprocesses oder des dabei wahrnehmbaren Fötors verantwortlich gemacht, bald aber fallen gelassen worden. Ein hervorragendes und andauerndes Interesse kommt allein dem von $\mathbf{A B E L}{ }^{1192}$ so genannten $\mathbf{B}$ a c i l l u s $\mathrm{mucosus} \mathrm{oza}$ e $\mathrm{na}$ e zu. Dieser Bacillus ist zuerst von LöwenBerg ${ }^{1144} \mathrm{im}$

Ozänasecret häufig gesehen und als Urheber des Processes angesprochen worden, freilich auf Grund bakteriologisch unzulänglicher Unterlagen. Erst die späteren Arbeiten von ABeL ${ }^{1192}$, Patrsen ${ }^{1197}$, Löwenberg ${ }^{1198}$, Fricke ${ }^{119}{ }^{9}$ und Stein ${ }^{1193}$ lehren uns den Bacillus genau kennen.

Er ist dem $\mathrm{Bacillus}$ p n u mo n a e Friedränders sehr nahe verwandt. Nach Fricke ${ }^{1199}$ stellen beide nichts anderes alsVarietäten des B a cill u s $\mathrm{mucosus}$ capsulat us dar, die lediglich veränderten Lebensbedingungen ihre Differenzirung verdanken. Noch weiter gehen KLEMP Eirer \& ScheIER 1104. Auf Grund von Immunisirungs- und serodiagnostischen Experimenten sehen sie sich genöthigt, das Frtedländersche, das Abelsche und das R h in os kl e r o mb a c t e r i u m für völlig identisch mit einander zu erklären. Diese Behauptung hält der neueste Bearbeiter der Frage, STREIT ${ }^{1195}$, für nicht hinreichend bewiesen. Aber auch er findet, daß das FrIEdLändersche und das Sklerombakterium durch Cultur und thierpathogenes Verhalten nicht von einander zu trennen sind.

1192. AвeL: Die Ätiologie der Ozäna. Z. f. Hyg. 21. 1895. 1193. Streir, W.: Zur Bakteriologie der Ozäna. Cbl. f. Bakt. 1. Abth. Bd. 28. 1900. 1194. KLeMPERer, F. \& Scheter: Üb. die Identität der Ozäna- u. d. Rhinosklerombac. mit Friedü̈nderschen Bacillen. Z. f. klin. Medic. 45. 1195. Streit, H.: Weitere Beitr. z. Sklerom, insbes. z. Frage: Gibt es Unterschiede zw. Sklerom- u. Frifdï̈nder-Bazillen etc. ? A. f. L. 19, S. 408.1907. In diesen Arbeiten findet man a u führliche Literaturangaben. 1196. LaUtmann: Ist die genuine Ozäna eine Infectionskrankheit? Z. f. L. 1, S. 421. 1908. (Kritik dor phantastischen Behauptungen von Perez 1175, der seinen aus dom Nasenschleim von Hunden stammenden „Cocco-bacillus foetidus ozaenae" als den Erreger der Ozäna ansieht.) 1197. PadLsen, E.: Ửber einen schleimbildenden Kapselbacillus bei atrofirenden Rhinitiden. Mitth. f. d. Verein schlesw.-holst. Ärzte. N. F. Jhg. 2, No. 1. 1198. LöwenBera: Le microbe de l'ozène. Ann. de l'inst. Pasteur. No. du 25. Mai 1894. 1199. Fricke: Üb. den sog. Bacillus mucosus capsulatus. Z. f. Hyg. 23. 1896. 
Der ABELsche Bacillus wird besonders in dem zähen Schleim gefunden, der zwischen Ozänaborke und Schleimhautoberfläche liegt (\$ 391), häufig in Reincultur, selten in so geringer Menge, daß es mehrfacher Secretentnalımen und Culturen bedarf, um ihn nachzuweisen. In die Schleimhant selbst dringt er nicht ein.

STR ÜBING ${ }^{1173}$ hat durch Übertragung des ABELschen Bacillus auf die Nasenschleimhaut eines schwindsüchtigen Todescandidaten einen Proceß erzeugen können, den er für Ozäna erklärt. Gegen diese Deutung protestiren, wie ich glaube mit Recht, KLemperer \& Scheiter. Dreyfuss \& Klemperer ${ }^{1200}$ erhielten bei 2 Inoculationsversuchen negative Resultate.

Auf die Bedeutung des ABetschen Bacillus für den OzänaproceB kommen wir noch zurïck (SS. $377,378,382 \mathrm{f}$.).

Ich habe mich bemüht, die wichtigsten Thatsachen über die Ozäna darzulegen und gehe jetzt dazu über, aus diesem Material heraus das 402 Wesen der Krankheit zu erörtern.

Dazu ist es nützlich, vorerst die hervorragendsten Symptome in ihrer Genese zu beleuchten, uns zu fragen: Woher stammt das zähe, zur Eintrocknung neigende Secret? Woher stammt der üble Geruch? Wie entsteht die Atrophie der Schleimhaut und des Nasengerüstes?

Woher stammt das zähe, zur Eintrocknung neigende Secret? Unsere Definition schließt circumscripte Entzündungen oder Eiterungen, besonders solche der Nebenhöhlen und der Rachenmandel aus, und damit ist schon gesagt, daß wir an eine Flächensecretion appelliren. Eine solche wollen mehrere Autoren ${ }^{1200 a}$ direct gesehen haben. GOTTSTEIN ${ }^{120}$ berichtet darüber: ,Allmählich bedeckt sich die Schleimhaut in eigenthümlicher Weise mit Secret, es erscheinen einzelne zerstreute graue Pünktchen, die Schleimhaut gewährt den Anblick, als wäre sie mit Mehlstaub bestreut. Indem nach und nach immer mehr solche Punkte erscheinen, bilden sie oft erst nach vielen Stunden eine Schicht, die die Schleimhaut gleichmäßig be deckt." GRÜNWALD ${ }^{1204 / 5}$ will diese und ähnliche Beobachtungen nicht gelten lassen. Er hat sie nämlich an einem eigenen Falle nachträglich als irrig erweisen können, indem er entdeckte, daß der in sehr dünner Schicht von oben, aus den Stirn- und Kieferhöhlenmündungen, herabfließende Eiter sich an einzelnen Schleimhautstellen staute und allein hier sichtbary wurde. Dagegen ist zu bedenken, daß HAJ ${ }^{1202}$ seine Beobachtung mehrere Jahre nach der Veröffentlichung der GrüNwaLDschen Kritik mittheilt (1899) und auch in der 3. Auflagese ines

1200. Dre yfuss \& Klemperer: Z. Bakteriol. d. Ozäna. 68. Vers. D. Naturf. u. Ärzte. 1896. Frankfurt. ref. Semons Cbl. 13, S. 106.1200 a. L. c. NoNo. 1201. 794. 1147. 1202. 1203. 1201. Gottstein: Nasenkrankheiten. Eulenburgs Realencyclopaedie. 1. Aufl. 1881. 1202. HaJeK: Path. u. Ther. d. entz. Erkr. d. Nebenh. d. Nase. 1. A. 1899. (3. A. 1909. S. 370.) 1203. Döвelf, E.: Üb. d. Bildung d. Secrets b. d. Ozäna. A. f. I. 15, S. 142. 1904. 1204. Gr ÜNWALD: Die Lehre von den Naseneiterungen. 2. A. 1896. S. $41 \mathrm{ff}$. 1205. Ders.: Der heutige Stand der Ozäna-Frage. A. f. L. 13.1902. 
Buches (1909) an ihr festhält, also gewiß gute Gründe haben muß, die von GRÜNWALD urgirte Täuschungsmöglichkeit auszuschließen.

Grünwald ist der Ansicht, da $B$ der $\mathrm{Oz}$ ä $\mathrm{n}$ a eiter stets ron circumscripten $H$ erden $\mathrm{komme}$, die nur wegen der mangelhaften diagnostischen Fertigkeit und Findigkeit der Untersucher nicht entdeckt würden. Zum Beweise führt er zunächst zahlreiche Fälle an, in denen es ihm geglückt sei, sog. Ozäna durch Aufdeckung und Ausheilung derartiger Herderkrankungen (Nebenhöhlen-, Rachenmandelaffectionen) dauernd żu beseitigen. Sodann ergebe ihm eine kritische Analyse der bisherigen Leichenuntersuchungen Resultate, die für seine Behauptung sprächen. Es seien nämlich nur in wenigen Fällen keine Nebenhöhlenempyeme auffindbar oder auszuschließen gewesen und, wo solche bestimmt negirt würden ( $\$ 399)$, wäre Rachenmandeleiterung wahrscheinlich. (Weiteres s. § 410).

Nun hat aber - trotzdem GR ÜNwald das Gegentheil versichert - E. FrïNKeL in seinen beiden Fällen von echter Ozäna doch über den Nasenrachen berichtet. In dem einen Falle ${ }^{1179}$ war außer einer bläulich-schwarzen Verfärbung der Mucosa in der Umgebung der rechten Tubenmündung der Nasenrachen völlig normal. In dem andern Falle ${ }^{1180}$ war die Schleimhaut im Nasenrachen, besonders am Dach und um die Tubenwülste herum, dunkel schiefrig verfärbt.

Es ist völlig ausgeschlossen, daß ein so gewissenhafter Beobachter wie E. FRÄNKEL bemerkenswerthe Veränderungen nicht beachtet oder nicht notirt haben sollte. Die Schleimhautverfärbung, über deren Natur sich F. nicht weiter äußert, hat gewiß in beiden Fällen nichts zu bedeuten. Es wird deshalb der zweite Fall ebenso wie der erste

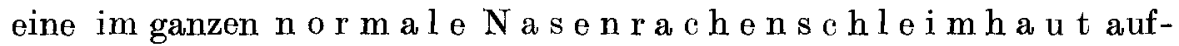
gewiesen haben, jedenfalls frei von solchen Veränderungen gewesen sein, die zur Entstehung des Ozänabildes führen könnten.

Daraus ergibt sich, daß es wenigstens zwei Fällevon a usgesprochener Ozäna gibt, in denen sicherlick keine Herderkrankungen vorhandenwaren.

Die GrÜnwaldsche Herdtheorie ist eine Erweiterung der von C. MICHEL ${ }^{792}$ zuerst im Jahre 1873 auf der Naturforscherversammlung in Wiesbaden ausgesprochenen und mit gewohntem Scharfsinn begründeten Ansicht, wonach dia Ozäna auf Eiterung in den Siebbeinzellen und Keilbeinhöhlen zurückzuführen sei. Diese Ansicht haben mit geringen Modificationen in neuester Zeit wiederum NOEBEL \& LöHNBERG ${ }^{1206}$ zu der ihrigen gemacht. BrEsGen, der sich zunächst GR ÜNWALD durchaus ansehloß ${ }^{1207}$, scheint inzwischen von der Herdtheorie wieder zurückgekommen zu sein ${ }^{1208}$.

1206. Noebel \& LöhnberG: Ätiologie und operative Radicalheilung der genuinen Ozänc. B. kl. W. 1900, 11-13. 1207. BRESGEN: Beiträge zư „Ozäna“"-Frage. M. m. W. 1894, 10/11. 1208. Ders.: Zur Entstehung des Bildes der Stinknase. Die ärztl. Praxis. 1902, 4. 
Die z äh e Consistenz des Secrets und seine Neigung zum Eintrocknen kann auf ursprünglicher Wasserarmuth, auf reichlicherer Wasserentziehung in der weiten Nasenhöhle, auf rəichlicherer Beimengung fester Bestandtheile (z. B. von Eiterzellen, Hornlamellen) oder endlich auf der Wucherung des zähen Schleim producirenden Abelschen Bacillus ${ }^{1205}$ beruhen. Wahrscheinlich sind mehrere dieser Ursachen gleichzeitig wirksam.

Woher stammt der üble Geruch des Secrets? Daß der Fötor an das Secret gebunden ist, haben wir schon gehört, ebenso, daß das Secret ursprünglich geruchfrei ist und erst bei seinem Verweilen in der Nasenhöhle stinkend wird. Daß hierbei bakterielle Zersetzungsvorgänge im Spiele sind, kann a priori als sicher angenommen werden.

Man hat früher die Ansicht ausgesprochen, faulige Zersetzung stagnirenden katarrhalischen Nasensecrets reiche hin, um den Ozänagestank zu produciren ${ }^{1152 / 69}$. Man findet aber zuweilen sehr weite Nasenhöhlen mit reichlichem schleimigeitrigem Nasensecret, das tagelang stagnirt, ohne den geringsten Geruch, am wenigsten einen ozänaähnlichen Föter anzunehmen; und kann sichaußerdem leicht experimentell von der Unrichtigkeit jener Annahme überzeugen. Brütet man nämlich frisch gewonnenes Ozänasecret und ebensolches katarrhalisches Nasensecret nebeneinander in der feuchten Kammer, so nimmt jenes sehr schnell dencharakteristischen Ozänagestank an, dieses niemals(FRESE $\left.{ }^{1150}\right)$.

Es muß also in dem Ozänasecret noch etwas Besonderes stecken, was zum Fötor führt. Und dieses könnte nur in zwei Dingen bestehen, entweder in einer veränderten $\mathrm{Zusammensetzung} \mathrm{des}$ Secrets oder in der Anwesenheit eines eigent ümlicben Z e r s etzung s e r r e g ers. Es wäre möglich, daß jeder dieser Factoren für sich allein zur Erzeugung des specifischen Fötors genügte, es könnte aber auch sein, daß beide sich dazu vereinigen müßten.

Von zahlreichen $\mathbf{T h}$ e ori e en, die auf Grund derartiger Erwägungen aufgestellt sind, müssen einige hier kurz referirt werden.

1) Krause ${ }^{1187}$, Réthi ${ }^{1147}$ und neuerdings Frese ${ }^{1150}$ messen der fettigen 404

Herkunft des Fötors

(1)


ein von mir beobachteter Fall von isolirter $\mathrm{Oza}$ ena $\mathrm{trachea} \theta$ bei absolut gesunder Nase, gesundem Nasenrachen, Rachen und Kehlkopf bis zu den Stimmbändern, in dem die in der Trachea sitzenden dunklen Borken einen penetranten specifischen Ozänagestank ausströmten ${ }^{1209}$.

3) In der Metaplasiedes Cylinderepithels in verhornendes Pflasterepithel sieht Schuchardt ${ }^{115}$ eine wesentliche Vorbedingung für das Zustandekommen des Ozänagestanks. Er weist darauf hin, daß auch an andern Stellen des Körpers, wo Hornsubstanz in Zersetzung geräth, höchst widerliche Gerüche auftreten, wie bei den Schweißfüßen, den Achselschweißen, im Präputialsack, bei den Cholesteatomen, bei gewissen mit Epidermisirung des Epithels einhergehenden Entzündungen der Gebärmutterschleimhaut. SeIFERT ${ }^{1189}$ schließt sich Schuchardt an und stützt die Anschauung durch die Mittheilung, daß er in Fällen von einfacher Atrophie des Naseninnern ohne Fötor keine Epithelverhornung auffinden konnte. Gegen die Schuchardtsche Theorie spricht, daß E. FräNkEL in einem seiner Fälle ${ }^{1180 .}{ }^{1210}$ keine Epithelmeaplasie constatiren konnte.

4) Der ABELsche Bacillus erzeugt auf den künstlichen Nährböden keinen üblen, sondern einen angenehmen, aromatischen Geruch ${ }^{1150 / 92 / 98}$. Er kann also für den Foetor ozaenae nicht direct verantwortlich gemacht werden. Dagegen könnte er wol mittelbar dazu beitragen, indem er das zähschleimige Substrat lieferte (S. 377), das dem oder den eigentlichen bisher noch hypothetischen Gestankerregern als Nährmaterial diente.

Woher stammt die Atrophie der Schleimhaut und des Nasengerïstes? Die hierüber aufgestellten, sogleich zu referirenden Theorieen lassen sich in zwei Gruppen einordnen. Die einen nehmen an, daß die zur Atrophie führende Noxe von der Oberfläche der Schleimhaut her einwirke, daß also successive zuerst die Schleimhautoberfläche, dann die tieferen Schichten und schließlich die Knochenunterlage erkranke. In die zweite Gruppe gehören die Ansichten, nach denen von einer entfernteren Ursache her infolge einer Entwickelungs- oder Ernährungsstörung Schleimhaut und Knochen gleichzeitig erkranken sollen oder zuerst der Knochen und später das Integument.

1) Viele ${ }^{1211}$ betrachten die Atrophie als das Endstadium des Theorieen ehronischen Katar hs. Durch bindegewebige Umwandlung der Infiltrationszellen, durch Schwund von Drüsen und Gefäßen nähme dabei die Schleimhaut einen narbigen Charakter an, der von KraUSE ${ }^{1187}$ mit der Xerosis conjunctivae und mit den callösen Stricturen der Harnröhre, von Anderen ${ }^{1147}$ mit der cirrhotischen Schrumpfung der parenchymatösen Organe verglichen wird. Da an der Atrophie auch der periostale Theil der Nasenauskleidurg betheiligt sei, so dürfe es nicht Wunder nehmen, wenn auch der Knochen atrophire ${ }^{1180, ~ S . ~} 517$.

Warum aber läuft nur ein kleiner Theil der so überaus häufigen chronischen Nasenkatarrhe in Atrophie aus? Darauf erhält man keine befriedigende Antwort. Wenn nämlich von einer auf dem Boden der Dyskrasie (Skrophulose) wurzelnden Neigung zur Atrophirung gesprochen wird, so ist damit nichts gewonnen, und es

1209. Zarniko: Ozaena trachealis. D. m. W. 1895. Vereinsbeilage S. 124. und Lïbecker Naturforscherversammlung. Autoreferat. Semons Cbl. 1896, S. 88. 1210. FräNked, E.: Demonstration in der biolog. Abth. d. Hamb. ärztl. Vereins. 29. 6. 97. Münchn. med. W. 1211. u. A. B. FRÄNKEL ${ }^{791}$, GotTStein ${ }^{1146}$, Schä̈fler ${ }^{1178}$, KraUse ${ }^{1187}$, E. Frankel ${ }^{1180}$. ZUCKERKaNdl ${ }^{9}$, STRÜ̈ING ${ }^{1173}$. 
bleiben die Fälle unerklärt, in denen die Krankheit gesunde und blühende Personen befällt.

Und warum erkranken Weiber so viel häufiger als Männer, obwol der chronische Nasenkatarrh bei Männern häufiger angetroffen wird als bei Weibern ? ${ }^{1216}$

2) Der eben erwähnten Ansicht ist die von AвEL ${ }^{1192}$ geäußerte verwandt. A. glaubt, die vom Bacillus mucosus ozaenae producirten $\mathrm{G}$ if $\mathrm{t} \mathrm{s}$ t of $\mathrm{f} \mathrm{e}$ wären es, die in die Schleimhaut hinein diffundirend diese zur Entzündung und später zur Atrophie brächten. Daneben käme der sogleich zu besprechende Borkendruck in Betracht.

3) Schon vor vielen Jahren hatte WALB ${ }^{2145}$ den 1 ange Z eit an da uernden Druek der Ozänaborken für die Entstehung der Atrophie verantwortlich gemacht. Diese Ansicht hat neuerdings wieder Anhänger gefunden ${ }^{1161-63 .}{ }^{1173.1202}$. Der, ,wie ein Collodiumverband wirkende Borkenüberzug" ${ }^{1205}$ soll durch andauernde Compression des Schwellkörpers mit seinen auch den Knochen ernährenden Gefäßen neben Anderem eine Ursache der Atrophie abgeben. - Dagegen wendet ChorewA ${ }^{1148}$ ein, daß die Borkenbildung hauptsächlich in der Gegend der mittleren Muschel zu finden sei, die Atrophie hauptsächlich an der unteren. Muschel. - Es ist auch schwer verständlich, wie bei der ausgezeichneten Gefäßversorgung der Nasenschleimhaut, bei den zahlreichen Anastomosen von Arterien und Venen mit andern Gefäßgebieten ( $\$ 29$ ff.) Ausschaltung eines Bezirks eine Verkümmerung benachbarter Bezirke verursachen soll. Warum entsteht keine Knochenatrophie, wenn man die Schwellkörper der unteren Muscheln noch so ausgiebig durch Galvanokaustik zerstört oder sie abschneidet?

4) Nach seinen mikroskopischen Untersuchungen ist Condes ${ }^{1148}$ zu der Ansicht gelangt, daß es bei den fraglichen Knochenveränderungen nicht um entzündliche Processe, sondern um selbständige primäre Vorgänge sich handele. Cholewa ${ }^{1148}$ glaubt, daß diese Vorgänge mit der $\mathrm{O}$ s $\mathrm{t}$ e o $\mathrm{m}$ a $\mathrm{l}$ a $\mathrm{c}$ i e vergleichbar seien. Durch das Eingehen zahlreicher Markräume und Haversischer Kanäle werde der Nasenschleimhaut ein gut Theil ihrer arteriellen Zufuhr unterbunden. $\mathrm{H}$ eraus resultire Erschlaffung und Muskelschwund in den Schwellkörpern und weiterhin Veränderung der Secretion und Atrophie der Schleimhaut. - Auch die Theorie von CHorewa scheint mir die vortreffliche Blutversorgung der Nasenschleimhaut von den verschiedensten Seiten her nicht hinreichend in Betracht zu ziehen.

5) ZaUfar ${ }^{1152 / 69}$ hat behauptet, die Nasenweite bei der Ozäna sei eine Folge angeborener Atrophie der Nasenmuscheln. Gegen diese Anschauung wendet ZUCKERKANDL 9, S. 2:3 ff. ein, er habe bei vielen Hunderten von Neugeborenen und Embryonen niemals auch nur die Spur einer rudimentären Nasenmuschel vorgefunden und hält damit die Theorie Zaurars für widerlegt.

Allein HopMaNN ${ }^{1160 / 62}$ führten seine bereits referirten Messungen ( $\$ 393$ ) zu der Anschauung ZaUfals zurück. Er glaubt, daß man, um von congenitaler Atrophie zu sprechen, nicht ausgebildete atrophische Veränderungen beim Neugeborenen zu fordern brauche. Es könne sich um eine immanente Anlage handeln, die sich erst später im Verlaufe der Entwicklung des Schädels manifestire. In diesem Sinne seien die atrophischen Veränderungen bei der Ozäna a.ls primäre aufzufassen.

6) Stroker ${ }^{1212}$ fand außer der Atrophie von Nase, Rachen und Kehlkopf häufiger analoge Veränderungen in der Trachea, in den Bronchen, in den Lungen-

1212. Sticker: Atrophie und trockene Fntzündung der Häute des Respirationsapparates; ihre Beziehung z. Syphilis (Metasyphilit. Xerose im Bereich der Athmungsorgane). D. A. f. klin. Med. 57. 1896. 
alveolen, ferner trockene Pleuritis, in manchen Fällen Atrophie im Bereich der Intestinalschleimhaut, einige Male auch eine Hautdegeneration in Form der Ichthyosis simplex oder diffusen Keratosis. Er vereinigt alle diese Erscheinungen

Xerose zu einem Krankheitsbilde, das er X e ros e nennt. „Die Xerose kann aus einzelnen Theilen des Respirationsapparates und wol auch andrer Apparate das eine und andre herausgreifen oder wenigstens deutlicher betheiligen als die uibrigen, oder auch das ganze System im Zusammenhange ergreifen. Thr Lieblingssitz ist der Nasenrachenraum." Als Hypothese fügt er hinzu, daß die Xorose in lotzter Iinie auf angeborene oder erworbene Syphilis zurückzuüfhren sei. Sie sei analog der Tabes ein "metasyphilitischer" Krankheitsproceß.

Freudenthal ${ }^{1213}$ und Blumenfeld ${ }^{1214}$ weisen auf die Rolle übermäßig trockener Luft für die Entstehung der Xerose hin.

409

Ozänatheorieen

Historisches

Begruin-

ding

inserer

Definition

Theorieen über das Wesen der Ozäna. Historisches ${ }^{1215 / 16}$. In früheren Zeiten bezeichnete man jede mit stinkender Absonderung verbundene Nasenerkrankung als Ozäna. Allgemein glaubte man, daß einer solchen immer Geschwüre oder Nekrosen zu Grunde lägen, und unterschied deshalb nach der Ursache, die man für die Ulceration auffand oder annahm, eine $\mathrm{Oza}$ ena syphilitica, tuberculosa, serophulosa ete.

Dieser Lehre trat zuerst Mrсhes auf der Naturforscherversammlung in Wiesbaden (1873) entgegen (S.376). Er behauptete, es gäbe eine nicht ulceröse Ozäna, ja diese sei weit häufiger als die ulceröse. MrcneL fand damals lebhaften Widerspruch. Bald darauf $\mathrm{kam} \mathrm{Z}_{\mathrm{AUFAL}}{ }^{1152}$, wie es scheint unabhängig von MICHEL, zu demselben Schlusse und seitdem hat die neue Lehre immer mehr Anhänger gewonnen und lange Zeit unbestritten das Feld behauptet. Zugleich gewöhnte man sich daran, die Bezeichnung Ozäna für die nicht uleerösen, fötiden Erkrankungen zu reserviren und es ist ein Verdienst B. FräNkels, die erste präcise Definition der Ozäna in diesem Sinne geliefert zu haben 791 (1 A. 1876).

Der Fortschritt, den man so erreicht zu haben glaubte, ist indessen seit einiger Zeit wiederum in Frage gestellt worden, und zwar durch GRÜNWALD 1204/5 (\$ 403).

Hatte schon MicheL ${ }^{799}$ die Ozäna auf eine Eiterung in den hinteren Nasennebenhöhlen zurückgeführt, so erweiterte GR ÜNwaLD diese Anschauung, indem er ganz allgemein Herdeiterungen im Bereich der Nase und des Nasenrachens für das Ozänakrankheitsbild verantwortlich machte. Derartige Herde seien zwar häufig in den Nebenhöhlen gelegen, sie brauchten es aber nicht immer zu sein. Sehr oft seien es z. B. die Rachenmandeleiterungen, auch syphilitische Schleimhaut- und Knochengeschwüre kämen in Betracht.

1213. Freudenthax, W.: Die Ätiol. d. Ozäna. A. f. 1. 14, S. 409. 1903. 1214. Buumenfeld, F.: Z. Ätiol. u. Ther. d. Xerosen d. Halsschleimhäute. ibd. S. 476. 1215. FräNkel, B.: Die Entwicklung d. Lehre v. d. Ozäna. B. lar. Ges. 16. 11. 06. B. kl. W. 1906, 52: 1216. Hopnann: Sicheres und Cnsicheres üb. Ozäna. M. f. O. 1907, S. 255. (Dieser Artikel befindet sich in erfreulicher Übereinstimmung mit meinen Ausführungen in der 2. Aufl. dieses Buches.) 
Aus den circumscripten Eiterungen leıtet Gr ünw al d das Ozäna bild auf folgende Weise ab: Das Secret werde durch Fäulniß stinkend. Dieser Vorgang werde begünstigt durch Retention des Secrets entweder am Orte der Entstehung oder nachdem es in die Nasenhöhle gelangt sei. Das stagnirende Secret nähme eine zähe Consistenz an theils durch Eintrocknung, theils durch Thätigkeit des ABELschen Bacillus, der darin eine ihm zusagende Brutstätte fände. So käme es zur Krustenund Borkenbildung. Es käme ferner durch den Druck der Borken $(\$ 407,4)$ zur Atrophie der Schleimhaut und schließlich auch des Knochengerüstes und die so hervorgerufene Weite der Nasenhöhle begünstige wiederum Stagnation, Eintrocknung und fötide Zersetzung des Secrets.

So gelangt GRÜnwaLd zu dem Schlusse: $\mathrm{D}$ i e $\mathrm{Oz}$ ä $\mathbf{n}$ a is t keine Krankheitsui generis, sie ist ein Symptomenbild, das durch Herderkrankungen verschiedenster Herkunft und verschiedenster L ocalisation hervorgerufen werden kann. Und er stellt sich damit wieder auf den ursprünglichen Standpunkt, der inzwischen von allen aufgegeben war.

Niemand wird sich dem bedeutenden Eindrucke der Ausführungen 411 Grünwalds entziehen können und Jedermann wird dankbar die Förderung anerkennen, die sie der Ozänafrage gebracht haben. Trotzdem hat es die übergroße Mehrzahl der Rhinologen abgelehnt, seine letzten Consequenzen gutzuheißen. Sie halten vielmehr an der Behauptung fest, daß es eine Ozäna ohne Herderkrankung gebe und daß von dieser rechtmäßigen Ozäna ( $O$. v e r a s. g e n u i n a) die durch Herderkrankungen erzeugten stinkenden Naseneiterungen abgetrennt werden müßten.

Die Gegner der Herdtheorie stützen sich dabei auf die Erfahrung, daß es in einem Theil der Fälle trotz eifriger und sachkundiger Bemühungen nicht gelingen will, Erkrankungsherde aufzufinden.

Dagegen sagt GRÜNwaLd: Daß die Herde nicht gefunden werden, beweist nicht, daß sie fehlen; es beweist nur, daß die diagnostische Fertigkeit des Untersuchers nicht hinreicht, um sie aufzudecken.

Diesen Einwand wird Jeder als berechtigt anerkennen, der die großen Schwierigkeiten der Erkennung mancher Nebenhöhlenaffectionen erwägt. Sehr häufig treten auch äußerliche Hindernisse (Ängstlichkeit, Zeitmangel des Patienten) einer vollkommenen Ausnutzung unsrer diagnostischen Mittel in den Weg. Es braucht deshalb in dem Einwurf kein Tadel erblickt zu werden, sondern vielmehr ein neuer Ansporn, unsre diagnostischen Fähigkeiten aufs Äußerste zu steigern.

Mir persönlich ist die Aufdeckung von Herderkrankungen stets dann mißlungen, wenn ich neben oder vielmehr in dem ausgeprägten Bilde der Ozäna, (Atrophie, Borkenbildung) den specifischen Fötor wahrnehmen konnte. Mit einer einzigen Ausnahme. Allein hier handelte sichs um einen Fall von com- 
binirten Empyemen der Kiefer- und Keilbeinhöhlen, der vorher anderwärts mit chemischen und thermischen Ätzungen gräulich mißhandelt war.

Übrigens scheint es hierzulande wenig Ozänen zu geben. Ich habe sie in den letzten Jahren ïberhaupt nur ganz vereinzelt gesehen und bin deshalb leider nur unvollkommen im Stande, der sehr begründeten Forderung Grünwalds nachzukommen, man solle es vermeiden, die Ozänafrage retrospectiv zu behandeln.

Eine viel sicherere Auskunft als die Untersuchung am Lebenden, muß in der Herdfrage die Leichenuntersuchung liefern. Hier hat die Kritik GR ÜNWALDS 1204, s. 53,'ff. zahlreiche Lücken und Ungenauigkeiten sowie falsche Werthung früherer Secretionsergebnisse nachgewiesen. Aber nach dem früher Gesagten ( $\$ 403$ ) bleiben doch die beiden Befunde E. FräNkeLs übrig und ebenso 2 Fälle OpPIkoFers ${ }^{1185}$, in denen sicherlich keine Herderkrankung vorhanden gewesen ist.

Ưbrigens leugnen die Gegner der Herdtheorie nicht das gelegentliche oder auch häufige Vorkommen von circumscripten Erkrankungsherden bei der Ozäna. Aber sie betrachten diese als complicirende Erkrankungen, die mit der Entstehung der Ozäna nichts zu thun hätten, es sei denn, daß z. B. eine Nebenhöhle in den Ozänaproceß mit einbe$z$ ogen würde.

Nach Ausschluß der Herderkrankungen gelangt man zu der Definition der Ozäna, von der wir ausgegangen sind.

Die sich mit dieser Ozaena vera s. genuina beErklärungs$\mathrm{f}$ a s s e $\mathrm{n}$ d e $\mathrm{n}$ Erklärungsversuche laufen alle darauf hinaus, die hauptsächlichsten Symptome, Atrophie und Absonderung eines (specifisch) fötiden, zur Eintrocknung tendirenden Secrets, entweder von einander oder von einer gemeinschaftlichen dritten Ursache abzuleiten. Sie sind in nuce in den früheren Angaben über diese Symptome enthalten und es wird deshalb genügen, mit wenigen Worten das Zusammengehörige zu verknüpfen. Einige dabei unvermeidliche Widerholungen möge der Leser nachsichtig mit in den Kauf nehmen.

1) Eine weitverbreitete auf B. FränkEL ${ }^{791.1215}$ zurückzuführende Theorie besagt, die Ozäna sei das Endstadium eines chronischen, hypertrophischen Katarrhs, weshalb ihr die von GotTstein geprägte Bezeichnung $\mathrm{R}$ hinitis at rophi c a $\mathrm{n}$ s f o e t id a zukomme. Der Gestank entstehe durch bakterielle Zersetzung des in seiner Zusammensetzung auf verschiedene Weise veränderten Secrets. Die gegen diese Theorie zu erhebenden Einwendungen ( $\$$ 397. 406) sollen hier nicht noch einmal vorgetragen werden.

2) Als eine reine $\mathbf{I}$ fectionskrankheit betrachten ABEL ${ }^{1192}$, Str ÜBING ${ }^{1173}$ und LerMoyez ${ }^{1217}$ die Ozäna. Nach der Ansicht der beiden Erstgenannten ist der ABELsche Bacillus der Erreger. Er erhalte unter gewissen Umständen, vielleicht dann, wenn die Nasenschleimhaut durch acuter oder chronischen Katarrh alterirt ist, die Möglichkeit zu haften. Er erzeuge ebenso wie in der künstlichen Kultur einen zähen Schleim, zu dem sich eitriges Nasensecret. geselle, um das zur Eintrocknung und Borkenbildung tendirende Ozänasecret zu liefern. Dieses Secret sei noch nicht fötid, es werde es erst, wenn einfallende Fäulnißkeime es zur Zersetzung brächten. Die Atrophie entstehe theils durch

1217. Lfermoyez, M. : La contagion de l'ozène. B. kl. W. 1906, 47. 
Secretdruck, theils durch Giftstoffe, die die an der Schleimhautoberfläche wuchernden Bacillen lieferten. - Der Proceß könne zunächst auf kleine Stellen beschränkt sein, er breite sich aber durch "Selbstinfection" sowol nach der Fläche als auch sprungweise nach den tieferen Luftwegen hin aus. - Gehäuftes Vorkommen entstünde durch directe Contagion.

Ihren Gedankengang logisch weiterverfolgend gelangen ABEL und StR ÜBING dazu, als $O z a ̈ n a j e d e S c h l e i m h a u t a f f e c t i o n d e r ~ N a s e z u$ bezeichnen, die einer activen Thätigkeit des Bacillus mucosus Abel ihre Entstehung verdankt, ob sie mit Fötor und Atrophie einhergeht oder nicht.

Leider hat die ansprechende Lehre AbELs durch die Untersuchungen von Fricke ${ }^{199}$ und besonders von KLfemperer \& SCHEIER ${ }^{1194}$ einen harten Stoß erlitten. Wenn es wahr ist, daß der Bacillus AbELs mit dem FriedLänderschen und dem $\mathrm{Rh}$ inosklerombacillus identisch ist ( $\$ 401$ ), dann muß man sich zu der Ansicht bekennen, er sei nicht die Ursache der Ozäna und des Skleroms sondern ein Saprophyt, der bei diesen Affectionen besonders günstige Ernährungsbedingungen vorfindet 1194 .

3) ZaUfaL ${ }^{1152 / 69}$ sieht angeborene Kleinheit der Muscheln als das Primäre 414 an. Bei der hierdurch bedingten übermäßigen Nasenweite hätte der Exspirationsstrom beim Schnäuzen nicht die zur Austreibung des Secrets nothwendige Kraft. Es bleibe in der Nasenhöhle zurïck, stagnire und gerathe durch hinzukommende Bakterien in putride Zersetzung. Die Unhaltbarkeit dieser letzten Behauptung ist schon dargethan ( $\$ 404$ ), auch hervorgehoben worden, daß Hopmans und in noch weiterer Ausdehnung STICKer sich der von ZuckerKANDL bekämpften Behauptung primärer Atrophie angenommen haben ( $\$ 408$ ).

STICKER betrachtet als letzte Ursache der Nasenatrophie und damit der Ozäna Syphilis, er hält die Ozäna für eine metasyphilitische Erkrankung. Schon vorher hatte GERBer ${ }^{1216}$ die beiden Affectionen mit einander in Verbindung gebracht, indern er die Ozäna in vielen Fällen als eine Manifestation der hereditären Spätsyphilis ansprach, und auf einen ähnlichen Standpunkt stellt sich neuerdings Frese ${ }^{1219}$, wenn er sagt, die locale luische Erkrankung der Nase lege häufig in frühester Jugend den Grund zu der wahrscheinlich allmählich noch fortschreitenden Atrophie. - In neuester Zeit hat man den Zusammenhang zwischen Lues und Ozäna auf serologischem Wege zu erforschen versucht 1220-21a. Dabei hat sich herausgestellt, daß die WassermanNsche Reaction bei sämtlichen daraufhin untersuchten Ozänakranken negativ ausfiel, was gegen die luische Provinenz der Ozäna spricht.

HopMaNN 1160/62 glaubt, daß mit der Atrophie des Gerüsts eine verminderte Widerstandsfähigkeit der Schleimhaut verbunden sei, die es leicht zu eitriger Rhinitis und weiterihn, durch Zersetzung des Secrets, zu fötider Rhinitis kommen lasse.

Hier kann auch auf die Ausführungen Särgens ${ }^{326-26}$ hingewiesen werden, die die Beeinträchtigung der Circulation bei zu weiter Nase behandeln ( $\$ 115)$.

1218. Gerber, P. H.: Spätformen hered. Syphilis i. d. oberen Luftwegen. 1894. Auch SтörK wäre hier zu nennen. Aber seine Ausführungen über dieses Thema sind so verworren, daß es mir bis jetzt nicht geglückt ist, ihren Sinn zu erfassen. 1219. Frese, O. : U̇̉b. d. Bez. d. Syph. z. Ozäna. A. f. L. 20, S. $4 \check{\jmath} 9$. 1908. 1220. Alexander, A. (Berlin): Serodiagnostische Unters. z. Frage d. Beziehungen zwischen Ozäna u. Syphilis. Z. f. L. 1, S. 669. 1909. 1221. ErsenLOHR, E.: Die Wassermannsche Reaction bei Ozäna. Z. f. O. 57, S. 401. 1909. 1221a. Sobernheim, W.: Wassermannsche Complementbindungsmethode $u$. Ozäna. A. f. L. 22, S. 1. 1909. 
Diese Circulationsstörungen sollen nicht allein eine Schwächung der Schleimhaut erzeugen (wie sie HopManN postulirt), sondern auch eine der Zersetzung günstige Veränderung des Secrets.

4) Den Anschauungen Hopmanns und Stickers nahe verwandt ist eine von $Z_{\text {ARNIKo }}{ }^{813,1209}$ ausgesprochene Hypothese: die Ozäna beruhe auf einer neurotrophischen Störung. Diese sei die primäre Ursache für die Atrophie der Schleimhaut und des Gerüstes. Die in ihrer Widerstandsfähigkeit geschwächte Schloimhaut reagire auf die gegen sie eindringenden Insulte mit Metaplasie des Cylinderepithels in verhornendes Pflasterepithel, und aus der Zersetzung des von der veränderten Schleimhaut gelieferten veränderten Secrets entstehe der specifische Fötor, vermuthlich durch Einwirkung eine ubiquitären Mikroorganismus, der in dem eigenthümlich zusammengesetzten Secrot einen adäquaten Nährboden fände.

Bald darauf äußerte sich BAYER ${ }^{1222}$ auf Grund der günstigen Beeinflussung, die ihm die sog. euprische Elektrolyse ( $\$ 421$ ) bei der Ozäna zu ergeben schien, und wie es scheint, ohne meine Ausführungen zu kennen, in ähnlichem Sinne.

BAYER schreitet von sehr unsicheren Grundlagen zu sehr sicheren Behauptungen fort. Ich finde nicht, daß der Sache damit gedient ist, halte es vielmehr für richtiger, über das Hypothetischeder ganzen Ann a h mekeinen $Z$ weif el a $\mathbf{u}$ fommen zu lassen. Ich habe mich auch - was moine Gegner zu übersehen scheinen - stets unzweideutig in diesem Sinne ausgesprochen.

Mir war es vor Allem darum zu thun, für meine Überzeugung, $\mathrm{d}$ a $\beta \mathrm{d}$ i o Ozäna nieht auf einer ektogenen, sondern auf einer endogenen, primären Ursache beruhe, ein Schlagwort zu finden; die Ozäna als eine vom chronischen Nasenkatarrh abzutrennende Krankheit sui generis hinzustellen; und von einem einheitlichen Grunde aus das gesamte Krankheitsbild ungezwungen abzuleiten.

5) Wir haben gehört, daß CHoLew a ${ }^{1148}$ die Atrophie der Nasenschleimhaut von einer primäre Erkrankung der Knochen des Nasengerüstes, einer Art von Osteomalacie dieser Knochen, ableitet ( $\$ 408)$. Die unmittelbaren Folgen des hieraus hervorgehenden veränderten Füllungszustandes der Schwellkörper seien, so deducirt er weiter, Veränderungen der Secretion. Das Secret werde stärker alkalisch und es werde langsamer abgesondert. Menge sich diesem Secret Eiter bei, wie ihn die Oberfläche der entzündeten Schleimhaut producirt, so entständen die richtigen Ozänaborken und durch deren Zersetzung der Gestank.

6) Hatten schon SchuchardT ${ }^{157}$, SeIFERT ${ }^{1189}$ und ZarNIKo ${ }^{813}$ der Epithelmetaplasie bei der Ozäna eine hervorragende Bedeutung zuerkannt ( $\$ \S 400.405$ ), so betrachtet SiEBENMANN ${ }^{1223 / 24}$ sie als das Wichtigste und Ausschlaggebende, sodaß soin Schülor Minder ${ }^{1182}$ sagt: „Bei der Umgrenzung des Begriffes Ozäna ist der mikroskopische Befund in den Vordergrund zu stellen (Siebenmans) als absolut sichores diagnostisches Moment und nicht der Fötor, noch die Muschelatrophie." Die Metaplasie des Epithels betrachtet $\mathbf{S}$. nicht als erworben, sondern als angeboren. Sie allein erzeuge aber noch keine Ozänæ. Diese entstehe erst, aus der ,latenten“ Ozäna werde eine „manifeste“, wenn zu der Epithelveränderung eine abnorme Weite der Nasenhöhle trete, wie sie besonders als eine Theilerscheinung der Chamäprosopie ( $\S 109)$ vorkomme ${ }^{1225}$. Die Weite der Nasenhöhle

1222. Bayer: Üb. Ozäna, ihre Ätiologie u, ihre Behandl. vermittels d. Elektrolyse. M. m. W. 1896, 32/33. 1223. Siebenmann: Nasenhöhle u. Gaumenwölbung bei den verschiedenen Gesichtsschädelformen. W. m. W. 1899, 2. 1224. Metsser (aus Siebenmanns Klinik): Chamaeprosopie, ein ätiologisches Moment für manifeste Ozäna (Rhinitis atrophica foetida). A. f. L. 8. 1898. 1225. Bestritten von SchönemanN ${ }^{1141}$. 
bewirke durch Vermehrung der Luftzufuhr, daß die Schleimhaut mehr den Charakter der Epidermis annehme. Die Epithelschicht werde dicker, die oberflächlichsten Zelllagen verhornten. Die Gefäße würden zum großen Theil verödet und es schwände der Knochen, soweit er von diesen Gefäßen ernährt werde. Alle diese Veründerungen vergleicht $\mathrm{S}$. mit analogen Vorgängen beim Cholesteatom des Mittelohres.

Auf diese Weise werde es verständlich, daß Epithelmetaplasie zwar sehr häufig in den engen Nasenhöhlen der Leptoprosopen und bei einseitiger Ozäna auf der engeren Seite gefunden werde, daß es hier aber nicht zum ausgeprägten Bilde der manifesten Ozäna käme.

Aus der Zusammenstellung der hauptsächlichsten Ozänatheorieen ersieht man, wie verschiedenartig sich das Bild der Krankheit in den einzelnen Köpfen malt. Wer wollte heut entscheiden, welche Ansicht die richtige ist?

Diagnose. Nach dem früher Gesagten ( $\$ 411)$ müssen wir mit de ${ }_{i}$ Diagnose Ozäna sehr zurückhaltend sein, sie erst aussprechen, wenn mit Sicherheit circumscripte Erscheinungen, insbesondere Nebenhöhlenempyeme und Rachenmandelaffectionen ausgeschlossen oder als lediglich complicirende Erkrankungen dargethan werden können. Daß das gewöhnlich mit den allergrößten Schwierigkeiten verknüpft ist, sei hier noch einmal hervorgehoben.

Über die diagnostische Bedeutung des Foetor ozaenae sind die Ansichten getheilt. Ich halte ihn wie viele andere für specifisch ( $\$ 390)$ und er ist mir ein wichtiges diagnostisches Zeichen (\$ 411).

Nach meiner Erfahrung sind Ozänakranke viel häufiger anosmisch als Nebenhöhlenkranke. Diese werden von etwaigen intranasalen Foetores ganz zuerst und viel mehr belästigt als ihre Umgebung; bei der Ozäna ist es umgekehrt: die Kranken verbreiten einen üblen ihre Umgebung im höchsten Grade belästigenden Geruch, empfinden ihn aber selbst nicht.

Von allen Untersuchern, die ihm ihre Aufmerksamkeit zugewandt haben, ist der ABELsche Bacillus ganz gewöhnlich in reichlicher Vermehrung unter den Ozänaborken angetroffen worden. Man kann dieses Verhalten, auch wenn man der ABELschen Theorie nicht beipflichtet, doch zur Differentialdiagnose verwerthen ${ }^{1148}$ und es wäre um so bedeutungsvoller, wenn sich als sicher herausstellte, daß der Bacillus in sonstigen eitrigen und borkigen Nasensecreten nicht oder viel weniger gut gedeiht, als im Ozänaeiter.

Einigen Anhalt liefert auch der Erfolg der Therapie. Eine uncomplicirte Ozäna verliert bei zweckmäßiger Behandlung mit GOTTSTEINschen Tampons in Kurzem ihren Gestank; stinkende Sinusempyeme oder Knochennekrosen werden dabei wenig oder gar nicht beeinflußt.

Wir werden später hören, daß das $\mathrm{Sk} l$ e r o m der Nasen- und Rachenschleimhaut in seinen ersten Stadien ein der Ozäna sehr ähnliches klinisches Bild geben kann. In solchen Fällen können nur die histologische Untersuchung und der weitere Verlauf die Diagnose sicherstellen. 
Prognose. Eine Ausheilung der Ozäna in dem Sinne, daß die atrophischen Theile ihre frühere Ausdehnung und Zusammensetzung widererhielten, gibt es nicht. Dagegen sind, wie schon mitgetheilt, einzelne Fälle beobachtet, in denen spontan, andre, in denen nach consequenter Behandlung ein Zustand eintrat, in dem die Patienten ohne therapeutische Proceduren dauernd oder längere Zeit von jeglichen Beschwerden (Borkenbildung, Fötor, subjectiven Störungen) frei blieben, sodaß man von einer Heilung in bedingtem Sinne sprechen konnte. In jedem Falle ist es möglich, die Erkrankung zu cachiren, indem wir Borken und Fötor wegbringen und die Kranken in den Stand setzen, sich in diesem Zustande dauernd zu erhalten.

Therapie. Hierzu stehen uns vor allem zwei Mittel zu Gebote, die GotTsteinsche temporäre Tamponade ( $\$ 204$ ) und die Nasendusche ( $\$ 207)$. Man kann vielfach mit jedem allein auskommen. Häufig empfiehlt es sich, sie mit einander zu combiniren.

Man beginne die Behandlung damit, daß man in die erkrankte Nasenhöhle, bei doppelseitiger Erkrankung in beide, Tampons einlegt. Entfernt man diese nach $1 / 4-1 / 2$ Stunde, so kommt der größte Theil der Borken in der Regel mit heraus. Was zurückbleibt, sitzt lose auf und läßt sich leicht mit der Gummipumpe wegspritzen, wenn man den Strahl direct darauf richtet, oder mit Sonde und Zange entfernen. Secretklumpen, die im Nasenrachen festsitzen, können nach Säuberung der Nasenhöhlen durch diese mit der Sonde gelockert und mit der Zange extrahirt werden, oder man geht vom Munde her mit einem Nasenrachenwischer (S. 208) ein und sucht das Secret loszubekommen, wobei gewöhnlich eine leichte Flächenblutung eintritt. Auf diese Weise gelingt es häufig gleich am ersten Tage Nase und Nasenrachen vollkommen zu reinigen, den Gestank zu bannen. Sicherlich ist dies aber am zweiten oder dritten Behandlungstage möglich. Inzwischen hat sich der Patient eine Nasenpumpe besorgt und man unterweist ihn gründlich in ihrer Anwendung. Insbesondere zeigt $\operatorname{man}$ ihm, wohin er das Ansatzstück zu richten hat, um die Prädilectionsstellen der Borkenbildung zu treffen. Ist der Patient in allem sicher, so besorgt er fortan die Reinigung selber, indem er morgens und abends die Dusche applicirt. In mehrtägigen Intervallen erscheint er zur Controlle. Finden sich dabei Borken vor, so forsche man nach der Ursache. Fast immer wird man einen Fehler in der Anwendung der Dusche finden. Ist das nicht der Fall, so hat der Patient die Dusche durch die Tamponade zu unterstützen. Man zeigt ihm die Zubereitung der Wattewieke und die Richtung, in der er sie einzuführen hat. Dazu bedient er sich am besten unsres früher abgebildeten Watteträgers (S. 206). Er legt die Tampons entweder morgens vor dem Duschen für 1 Stunde ein, oder am Abend abwechselnd in eine oder die andre Nasenseite, um sie die Nacht über darin zu lassen.

Als Duscheflüssigkeit reicht die physiologische Kochsalzlösung 
oder eines ihrer Surrogate (S. 204) gewöhnlich aus. Von desinficirenden Lösungen käme die Borlösung in Betracht, der man eine kleine Menge Wasserstoffsuperoxydlösung (einige Eßlöffel auf den halben Liter) zusetzen kann. Die übrigen sind entbehrlich und in den Fällen, wo noch etwas Geruch vorhanden ist, verwerflich. Die Hauptsache ist die mechanische Wirkung des Spitzenstrahls, die Reinigung der Nase, die es zu einer Eintrocknung und bakteriellen Zersetzung des Secrets gar nicht erst kommen läßt.

Hat man die Überzeugung gewonnen, daß der Patient mit Dusche und Tampon gut fertig wird, so kann man in immer längeren Zwischenräumen controlliren, wöchentlich, monatlich, vierteljährlich. Es darf dann auch die Reinigung seltener vorgenommen werden, einmal täglich, später einen Tag um den andern. Der Kranke ermittelt selbst am besten die ihm zuträgliche Dauer der Pausen.

In jedem Falle ist er von vornherein darüber aufzuklären, was wir mit unsrer Therapie leisten können und wollen. Er muß sich darauf gefaßt machen, die vorgeschriebenen Proceduren lange Zeit, vielleicht bis an sein Lebensende sorgfältig und unverdrossen auszuführen, wie er sich das Haar kämmt und die Zähne putzt, zu Hause und auf Reisen.

BRUCK 1226/27 will einigen Mängelnder GotTsteTNschen temporären Tamponade (daß sie zur Zeit immer nur auf einer Seite anwendbar sei, daß sie Eintrocknung und Krustenbildung nicht gänzlich verhindere) durch seine ,permanente Tamponade" abhelfen. Es wird ein passend zugeschnittener Streifen hydrophiler Gaze mit Hilfe einer englischen Bougie von angemessener Stärke in die Nase hineingestülpt, sodaß er wie eine Tapete den Nasenwänden anliegt. Sobald der Patient fühlt, daß die Gaze durchtränkt und gelockert ist, schnaubt or sie aus und ersetzt sie durch einen neuen Streifen. B. rühmt als Vortheile des Verfahrens, daß dadurch Eintrocknung und Zersetzung des Secrets mit absoluter Sicherheit hintangehalten werde und daß die physiologischen Functionen der Nase (Anfeuchtung und Erwärmung der Inspirationsluft) einigermaßen erfüllt würden.

KutTner 1156 hat, um die Gefahren der Nasendusche (\$208f.) zu vermeiden, einen Inhalationsapparat construirt, dessen warme Dämpfe die Lösung der Ozänaborken leicht und vollkommen bewerkstelligen sollen. $\mathrm{Zu}$ demselben $Z$ wecke erscheint mir der von LUBINSKr 1228 angegebene Inhalationsapparat empfehlenswerth.

Mit der bisher geschilderten, auf ausgiebige und dabei schonende 419 Reinigung des Naseninnern abzielenden Behandlung erreicht man alles bei der Ozäna überhaupt Erreichbare: Beseitigung von Borken und Fötor, wodurch die Kranken der menschlichen Gesellschaft zurückgegeben werden; Beseitigung der subjectiven Belästigungen; Unterstützung einer etwa vorhandenen natürlichen Heilungstendenz.

1226. Bruck, F.: Zur Therapie der genuinen Ozäna. B. kl. W. 1897, a. 1227. Ders.: Die Behandlung der genuinen Ozäna mittels der permanenten Tamponade. Allg. medic. Centralzeitung. 1899, 74. 1228. Lubinski, M. Z. Inhalationstherapie. D. m. W. 1909, 2. 
Doch hat es an Bemühungen nicht gefehlt, mehr als diese Ziele zu erreichen oder wenigstens, sie kürzer und vollkommener zu erreichen. Und die Zahl der hierfür empfohlenen Maßnahmen ist sehr groß.

Es liegt nicht in meiner Absicht, sie alle zu referiren. Ich will mich vielmehr mit einer summarischen, orientirenden U̇bersicht begnügen und Leser, die sich für die Einzelheiten interessiren, auf die übersichtliche und sehr vollständige Darstellung von GrosskopfF 1140 verweisen.

1) Man hat vielfach versucht, durch Application von d es inf i c ir enden oder desodorirenden $M$ itteln zu nützen, indem man die hypothetischen Infectionserreger oder die Zersetzungserreger bekämpfte. Es gibt wol kaum ein hierfür geeignet erscheinendes Mittel, das nicht angewandt und als wirkungsvoll empfohlen wäre.

So hat man Lösungen von Lysol, Ichthyol, Kalium permanganic. u. ä. als Spülwasser oder als Sprays auf die Nasenschleimhaut applicirt. Man hat diese und ähnliche Lösungen (z. B. Phenolum natrosulforicinicum Merck 30: 100 1229) mit der Wattesonde in die Nasenschleimhaut eingerieben. Andre blasen pulverförmige Medicamente auf, z. B. Argentum nitricum $(0,1-1,0: 10,0)$, Jodoform, Jodol, Aristol, Europhen, Nosophen, Kalium sozojodolicum, Zincum sozojodolic. (1: 10), Acid. citric.: Sacch. lact. $\overline{\text { aa }}$ (HAMM); noch andre bevorzugen Watteeinlagen, die mit den wirksamen Lösungen (Ammon. sulfoichthyolic. 30-40:100, Metakresolanytol 1-1,5:100, Kreolinvasogen 2:100, Mentholvasogen u. ä.) imprägnirt sind.

2) Um einestärkereDurchfeucht ungder Nasensehlei mh a ut, eine Verflüssigung und damit eine leichtere Eliminirung des Secrets zu erreichen, empfehlen einzelne Autoren die Darreichung von Jodkalium. L. WoLFE" (9. Vers. d. Vereins südd. Laryngologen) verordnet zu demselben Zwecke ganz. kleine Dosen ( $1 / 4-1 / 2$ g. p. die) von Jodnatrium.

3) Andre Maßnahmen haben eine Umstimmung der sehleimha u t zum Ziele. Von diesen ist als die mildeste die von SÄNGER ${ }^{326-28}$ befürwortete Einengung des übermäßig weiten Luftweges zu erwähnen, die, wie aus. den früheren Auseinandersetzungen abgeleitet werden kann, zu einer Verbesserung der Circulation und damit zu einer Kräftigung der Schleimhaut führen soll. S. erreicht das durch Einlegen einer Prothese, die aus zwei die Nasenlöcher theilweise zudeckenden für jede Nase zurechtzuschneidenden Metallplättchen und einem sie verbindenden Metallstreifchen besteht. Zu demselben Zwecke legt SprengEr. (B. kl. W. 1908, 46) Kügelchen von Gummischwamm in den Naseneingang. Viel complicirter ist der von KaFEManN (A. f. L. 2. S. 407) beschriebene Nasenobturator, der außer der Verengerung des Nasenluftweges die Inhalation flüchtiger desinficirender Medicamente (Menthol, Eucalyptol) gestattet. Endlich ist zu erwähnen, daß man neuerdings durch Paraffininjectionen das Volumen. der unteren Nasenmuscheln auf die Norm zu bringen versucht hat ${ }^{1230}$. Die

1229. Baumgarten: W. k. W. 1899, 35. Dreyfuss (Verh. d. Vereins. südd. Laryngologen, 8. Vers.) reibt das Mittel in die gründlich gereinigte Nasenschleimhaut, besonders in die Nischen und Winkel ein. Anfangs tëglich, später 3 mal, sodann $1 \mathrm{mal}$ wöchentlich. Der Fötor soll schon nach 2-3 maliger Einreibung verschwunden sein. Auch GrosskOPFF ${ }^{1140}$ bestätigt die günstigen Erfolge. 1230. Motre \& Brindel: Résultates des injections de paraffine faites sous la mouqueuse pituitaire chez 70 malades atteintes de coryza atrophique ozénateux-type. Le bull. médic. 23. 5. 1903. Brozcraert: Les injections de paraffine appliquées au traitement de l'ozène. Presse oto-laryngol. Belge 1903, 6. 
Methode dürfte zur allgemeineren Anwendung erst dann empfohlen werden können, wenn ihre Ungefährlichkeit in einer großen Anzahl von Fällen sicher bewiesen wäre.

Eine Umstimmung der Schleimhaut bezweckt auch die von mehreren ${ }^{757 / 8.1151}$ angewandte innere Vibrationsmassage.

Viel eingreifender wirken die von RÉTHI ${ }^{1147}$ gelobten Oberflächenätzungen der Schleimhaut durch Glühhitze oder durch chemische Mittel (Chromsüure, Trichloressigsäure) und ein heroisches, unter Umständen nicht ungefährliches Verfahren stellt die von BERTHoLD in die Rhinologie eingeführte Verbrühung der gesamten Schleimhaut mit strömendem Wasserdampf ( $\mathrm{V}$ a p o r is a $\mathrm{t}$ i o $\mathrm{n}$ ) $\operatorname{dar} 1231 / 2$.

Belgische Rhinologen (Cheval, Capart) haben die Elektrolyse in der besonderen Modification der cuprischen Elektrolyse (+ Kupfernadel in die mittlere Muschel, - Platinnadel in das Septum oder den unteren Nasengang; Stärke 10-15-30 M. A.; Dauer: ca. 10 Min.) begeistert gepriesen ${ }^{1233}$. Cheval verstieg sich zu der Behauptung, er hätte 91 proc. Heilungen, die meisten durch eine einzige Sitzung, erzielt. Man glaubte diese fabelhafte Wirkung einer baktericiden Kraft der elektrolytischen Zersetzungsproducte zuschreiben zu dürfen, wogegen BAYER 12a4, hauptsä̈hlich, weil er die Besserung nicht allein auf der behandelten Seite, sondern auch darüber hinaus im Nasenrachen und auf der andern Seite sah, aus dem Erfolge der Elektrolyse zu der Behauptung der neurotrophischen Natur der Ozäna gelangte ( $\S 415)$. Deshalb will BAYER der Elektrolyse die Bedeutung einer causaltherapeutischen Methode zuerkannt wissen.

4) Ähnliches prätendirt Cholewa ${ }^{1148}$ für die von ihm mehrmals geübte Infracturirung des atrophisohen unteren Muschelknochens. Sie soll wie ähnliche Eingriffe beim osteomalacischen Knochen, eine Ausheilung der Knochenerkrankung und damit der hiervon abhängigen Schleimhautveränderungen anbahnen.

Dem Beginnen Cholewas steht das Vorgehen Flataus 1235 nahe, durch Eintreibung von Elfenbeinstiften in den Muschelknochen eine Ausheilung der Krankheit zu Wege zu bringen.

5) Der Curiosität halber sei erwähnt, daß man Ozänakranken wahrhaftig Diphtherieheilserum - und natürlich mit Erfolg! - injicirt hat, weil in dem Ozänasecret häufig ein dem LöFfLERschen ähnlicher Bacillus gefunden worden ist ${ }^{1236}$. Dieser hat sich bei näherem Zusehen prompt als ein ganz gewöhnlicher Schmarotzer des Nasensecrets herausgestellt 1193.

6) In neuester Zeit hat man versucht, durch $\mathrm{H}$ y perämis ir ung der Schleimhaut nach BIER die Ozäna günstig zu beeinflussen. Es scheint, daß

Beide citirt von Furess: Die Behandlung der Ozäna mit Hartparaffininjectionen. B. k. W. 1904, 10 . BLAU, A.: Die Behandl. d. Ozäna mittels Paraffin-inject. A. f. L. 18, S. 448.1906 . Weleninsky: ibd. S. 548 . vgl. ferner Semons Cbl. 1905 ff. 1231. Berthold: Die intranasale Vaporisation. Berlin 1900. 1232. Boyé: Beiträge zur intranasalen Vaporisation. M. f. O. 1903, S. 213. 1233. Jahresvers. Belgischer Laryngologen u. Otologen. Brüssel 16. Juli 1895. ref. Semons Cbl. 1896, S. 406 ff. 1234. BAyer: Über Ozäna, ihre Ätiologie und Behandlung vermittels der Elektrolyse. M. m. W. 1896, 32/33. 1235. Flatad, TH. S.: Üb. zwei radicale Heilungen der Rhinitis atrophicans foetida durch eine neue operative Behandlungsmethode. Deutsche Praxis. 3, Heft 12. 1900. 1236. Belfanti \& della Vedova: Sull' etiologia dell' ozena ete. Giorn. d. R. accad. di Med. di Torino. 1896. 3. ref. Baumgartens Jahresber. 12. 1896. 
durch Luftabsaugung aus der Nase ${ }^{1237}$ eine wünschenswerthe Lockerung festaufsitzender Borken erzielt werden kann 1239. SPIEss 1238 spricht sogar von Dauerheilungen, doch bleibt die Bestätigung dieser Angaben abzuwarten.

Wenn man die Veröffentlichungen durchgeht, die die aufgezählten und die ihnen verwandten therapeutischen Maßnahmen zum Gegenstande haben, so wird man Folgendes bemerken: Die meisten haben vorzüglich in der Hand ihrer Erfinder und weniger andrer Autoren die erwarteten Resultate gegeben; und auch diese nur, solange die Behandlung dauerte.

Solche Mittel kann man natürlich nicht als Heilmittel bezeichnen. Man wird vielmehr zu der Vermuthung gedrängt, nicht die Proceduren selbst seien das Wirksame, sondern die mit ihrer Anwendung nothwendig verbundene Reinigung und Reinhaltung der Nasenhöhlen. Es wird doch beispielsweise Niemandem einfallen, ein Pulver oder eine medicamentöse Lösung in die ungereinigte, mit Borken ausgestopfte Nasenhöhle zu bringen. Auch vor der Vibrationsmassage und durch sie wird die Nasenschleimhaut gesäubert, das Secret verflüssigt. Und die Autoren, die über die glänzenden Erfolge der cuprischen Elektrolyse berichten, scheinen stets auch für eine ausgiebige Reinigung der Nase neben dieser Behandlung Sorge getragen zu haben. Läßt man nämlich die Reinigung fort, so bleiben auch die Erfolge aus ${ }^{1240 / 41}$.

Unter den Complicationen der Ozäna mögen hier die $\mathrm{N}$ e b e $\mathrm{n}$ h ö h l e n a f e c t i o n e n erwähnt sein. Sie sind nach später zu besprechenden Regeln einer geeigneten Behandlung zu unterwerfen.

Neben der localen ist die Behandlung des Allge meinz u standes nicht zu vergessen. Sie hat vorzüglich die Ernährung anämischer, skrophulöser Individuen zu berücksichtigen und die Kräftigung ihrer Gesamtconstitution anzustreben.

\section{Rhinitis (chronica) atrophica simplex ${ }^{790 / 94}$}

Zuweilen trifft man Personen mit auffallend weiter Nasenhöble und weitem Nasenrachen an.

In uncomplicirten Fällen kann jede abnorme Secretion fehlen und die Theile sehen dann genau so aus, wie die einer vortrefflich gereinigten Ozänanase. Häufiger aber findet man das Secret pathologisch verändert. Es ist weißlich, oder gelblich bis grüngelb, lagert gewöhnlich in etwas größerer Menge am Nasenboden, ist aber auch an höher gelegenen Stellen und am Schlunddache sichtbar und kann allenthalben zu Schalen und Krusten eintrocknen. Es ist entweder völlig geruchlos oder es weist einen geringen, faden Geruch auf, niemals aber den scheuslichen Foetor ozaenae.

Der Mesopharynx kann völlig intact oder im Zustande des einfachen chronischen Katarrhs sein oder er ist in den Proceß mit ein-

1237. Der vollkommenste Apparat dafür scheint mir der von HoRs angegebene zu sein (Z. f. O. 57, S. 24). 1238. SpIEss: Die therapeut. Verwendung d. negativen Drucks (Saugwirkung) b. d. Behandl. d. trockenen u. atrophischen Katarrhe d. Nase und des Rachens. A. f. L. 17, S. 179 . 1905. 1239. Sondermann: Weitere Erfahrungen mit meinem Nasensauger. ibd. S. 425. 1240. HENDELsонм: Ueb. Ozäna und ihre Behandl. mit cuprischer interstitieller Elektrolyse. M. f. O. 1897, S. 330 . 1241. HECHT: Zur therapeut. Verwerthung der Elektrolyse etc. A. f. L. 6.1897. 
bezogen. Es erscheint dann die Schleimhaut vollkommen glatt, wie gefirnist, die darunter sichtbaren Muskeln verleihen ihr die Farbe des rohen, geräucherten Fleisches (SCHECH ${ }^{805}$ ). Der dünne Überzug, der den spiegelnden Glanz erzeugt, läßt sich mit der Zange abtupfen oder mit einem Wattetupfer abwischen, wobei es leicht zu geringen Blutungen kommt.

Viel seltener als der Rachen sind die tieferen Luftwege vom Larynx abwärts in analoger Weise erkrankt.

Ulcerationen, Nebenhöhlenaffectionen fehlen.

Häufig soll eine Verminderung der Geruchsschärfe bis zur Anosmie vorhanden sein (JuRASz ${ }^{794}$ ).

Die Beschwerden der Kranken werden vorzugsweise durch die abnorme Weite der Nasenhöhlen ( $\$ 115)$ und ferner durch die Secretansammlung erzeugt. Sie bestehen in Trockenheitsgẹfühl in Nase und Hals, Neigung zum Räuspern, Schluckbeschwerden, Heiserkeit, erhöhter Empfindlichkeit gegen Temperaturschwankungen und Luftstaub.

Die Deutung des Krankheitsbildes hat stets von 424 seiner Ähnlichkeit mit der Ozäna ihren Ausgang genommen. Man hat sich entweder vorgestellt, daß die Krankheit die Vorstufe der Ozäna sei; oder, daß sie eine wirkliche Ozäna darstelle, bei der es nur mehr zufällig aus Mangel an Fäulnißerregern nicht zur Zersetzung und zur Gestankbildung käme; oder endlich, daß eine abgeheilte Ozäna vorläge. Über die Frage, ob die Atrophie eine secundäre oder eine primäre Erscheinung sei, herrschen dieselben Meinungsdifferenzen wie bei der Ozäna.

Bemerkenswerth ist der Befund von SEIFERT ${ }^{1189}$, den ich bestätigen kann, daß in den Fällen einfacher Atrophie Epithelmetaplasie nicht vorhanden, jedenfalls in weit geringerm Umfange vorhanden ist als bei der Ozäna. Dieser Befund ist neben andern für uns ein Grund gewesen, der Epithelveränderung eine Bedeutung für das Zustandekommen des Fötors zuzuerkennen.

In diagnostischer Hinsicht muß ebenso wie bei der Ozäna sorgfältige Berücksichtigung circumscripter Entzündungsprocesse, insbesondere von Nebenhöhlenaffectionen, gefordert werden.

Die Therapie hat vor allem für Reinhaltung der Nase und des Nasenrachens zu sorgen. Daneben erweisen sich für den Rachen massirende Pinselungen mit Jodglycerinlösung ( $\$ 381$ ) nützlich. Für die Nase scheint die Saugtherapie der Beachtung werth zu sein.

\section{Kapitel}

\section{Die infectiösen Granulome}

In diesem Kapitel behandeln wir eine Anzahl von Krankheiten - 425 Tuberkulose, Syphilis, Rotz, Lepra, Skleromdie wegen mannigfacher Analogieen zusammengehören. 